\title{
Identification of a basal stem cell subpopulation in the prostate via functional, lineage tracing and single-cell RNA-seq analyses
}

Xue Wang ${ }^{1,2}$, Haibo $\mathrm{Xu}^{3,4}$, Chaping Cheng ${ }^{1,2}$, Zhongzhong $\mathrm{Ji}^{1,2}$, Huifang Zhao ${ }^{1}$, Yaru Sheng ${ }^{1}$, Xiaoxia Li', Jinming Wang', Yu Shu ${ }^{1}$, Yuman He ${ }^{1}$, Liancheng Fan ${ }^{5}$, Baijun Dong $^{5}$, Wei Xue ${ }^{5}$, Chee Wai Chua ${ }^{1,5}$, Dongdong Wu ${ }^{3}$, Wei-Qiang Gao ${ }^{1,2,6 *}$ and Helen He Zhu' ${ }^{1,5,6 *}$

${ }^{1}$ State Key Laboratory of Oncogenes and Related Genes, Renji-Med-X Clinical Stem Cell Research Center, Ren Ji Hospital, School of Biomedical Engineering, Shanghai Jiao Tong University, Shanghai 200127, China;

${ }^{2}$ School of Biomedical Engineering \& Med-X Research Institute, Shanghai Jiao Tong University, Shanghai 200030, China;

${ }^{3}$ State Key Laboratory of Genetic Resources and Evolution, Kunming Institute of Sciences, $15 \quad$ Kunming 650223, China

${ }^{4}$ University of Chinese Academy of Sciences, Beijing 100049, China.

${ }^{5}$ Department of Urology, Ren Ji Hospital, School of Medicine, Shanghai Jiao Tong University, Shanghai, 200127, China

${ }^{6}$ Lead Contact.

20 Correspondence*: Wei-Qiang Gao (gao.weiqiang@sjtu.edu.cn) or Helen He Zhu (zhuhecrane@shsmu.edu.cn). Tel: 86-21-68383917, Fax: 86-21-68383916. Address: Stem Cell Research Center, Ren Ji Hospital, 160 Pujian Rd., School of Medicine, Shanghai Jiao Tong University, Shanghai, 200127, China. 


\begin{abstract}
The basal cell compartment in many epithelial tissues such as the prostate, bladder, and mammary gland are generally believed to serve as an important pool of stem cells. However, basal cells are heterogenous and the stem cell subpopulation within basal cells is not well elucidated. Here we uncover that the core epithelial-to-mesenchymal transition (EMT) inducer Zeb is exclusively expressed in a prostate basal cell subpopulation based on both immunocytochemical and cell lineage tracing analysis. The Zeb1 ${ }^{+}$prostate epithelial cells are multipotent prostate basal stem cells (PBSCs) that can self-renew and generate functional prostatic glandular structures with all three epithelial cell types at the single-cell level. Genetic ablation studies reveal an indispensable role for Zeb1 in prostate basal cell development. Utilizing unbiased single cell transcriptomic analysis of over 9000 mouse prostate basal cells, we find that $\mathrm{Zeb1}^{+}$basal cell subset shares gene expression signatures with both epithelial and mesenchymal cells and stands out uniquely among all the basal cell clusters. Moreover, Zeb1 ${ }^{+}$epithelial cells can be detected in mouse and clinical samples of prostate tumors. Identification of the PBSC and its transcriptome profile is crucial to advance our understanding of prostate development and tumorigenesis.
\end{abstract}

Key words: prostate basal stem cells, Zeb1, lineage tracing, single-cell RNA sequencing, Wnt signaling pathway, prostate cancer, tumor initiating cell, 


\section{Introduction}

The prostatic epithelium, comprised of basal cells, secretory luminal cells and rare neuroendocrine cells, can serve as an excellent model to study stem cell biology due to its ability to regress and regenerate after repeated rounds of androgen deprivation and restoration in mice and rats ${ }^{1,2}$. Using sub-renal capsule tissue regeneration, lineage tracing, cell division mode analyses or organoid-forming assays, we and others have demonstrated the existence of prostate stem/progenitor cells in both basal and luminal prostate epithelia ${ }^{3-16}$. However, none of the existing markers for basal stem cells (CD117, CD133, CD44, Trop2, CD49f, Sca1, etc.) ${ }^{8,15,16}$ is exclusively found in the basal cell compartment. Despite the long-term postulation that basal cells contain more primitive prostate stem cells due to their resistance to castration, capability to differentiate into basal, luminal and neuroendocrine cell lineages of the prostate epithelium, and susceptibility to oncogenic transformation $^{6,8,15,17-21}$, the identity and nature of the prostate basal stem cells (PBSCs) within the heterogenous basal cell epithelia have not been elucidated.

In the present study, we identify a PBSC subpopulation that expresses Zeb1, an important EMT inducer ${ }^{22,23}$, through both in vitro and in vivo functional, lineage tracing and genetic ablation analyses. Furthermore, we integrated single-cell RNA sequencing with computational data analysis, a powerful approach that is lately developed to map cellular diversity of a given tissue, i.e., cortical neurons ${ }^{24}$ and cancer related immune cells ${ }^{25}$, etc., to examine the prostate basal cell compartment. Our single-cell transcriptomics data provide additional supporting evidence for the existence of Zeb1-expressing PBSCs and uncover their gene expression profile. 


\section{Results}

\section{Zeb1 is exclusively expressed in a prostate basal cell subpopulation and more frequently detected in the urethra-proximal region}

EMT has been previously considered to occur only in early embryonic development or of core transcriptional factors such as Zeb1/2, Snai1/2, Twist1/2 which are required for EMT induction, have not been examined in normal prostate epithelia under physiological condition. Compared to prostate luminal cells, basal cells expressed low levels of epithelial-cell specific genes such as miRNA200 family $^{27}$ and E-cadherin ${ }^{3}$ and exhibited a more mesenchymal-like phenotype. We therefore asked whether core EMT inducers are present in normal prostate basal cells. Immunofluorescent analysis of prostate sections showed that less than $1 \%$ of basal cells were positively labeled for Zeb1 immunostaining (Fig. 1a, b). Snai1 or Slug (Snai2) was expressed in $20 \%$ or $90 \%$ of $\mathrm{p}^{+}$basal cells, respectively, while Twist $1 / 2$ positive staining was not detected in the basal layer (Fig. 1a, b and Supplementary Table 1). Considering the interesting expression pattern of Zeb1 in the prostate epithelium and the previously reported role of Zeb1 in stemness acquisition and maintenance of cancer stem cells (CSC) characteristics ${ }^{23,28,29}$, we decided to investigate the biological relevance of $\mathrm{Zeb} 1^{+}$basal cells in the prostate.

To better characterize and to more easily enrich the Zeb1 ${ }^{+}$prostate epithelial cells, we established a tdTomato knockin mouse model in which the coding sequence of tdTomato fluorescent reporter was linked to the last exon of Zebl by a P2A element (Fig. 1c). Using immunofluorescent co-staining of Zeb1 and RFP on mouse prostate sections, we confirmed that the tdTomato labeling faithfully reflected the endogenous expression of Zeb1 (Fig. 1d). TdTomato positive cells were only found in prostate basal cells (marked by CK5 immunostaining) but not 
from luminal cell compartment (labeled by CK8 immunostaining) (Fig. 1e). The Zeb1 $1^{+} /$tdTomato $^{+}$ prostatic basal cells were more frequently detected in the urethra-proximal region relative to the distal region, the location where prostate stem cells were suggested to reside ${ }^{8,30,31}$ (Fig. 1f, 1h and Supplementary Table 1). In addition, we found that the percentage of Zeb $1^{+} /$tdTomato $^{+}$basal cells declined from $2.2 \%$ at postnatal day $3(\mathrm{P} 3)$ to $1.0 \%$ at $\mathrm{P} 21$ as the prostate development proceeded, and decreased to $0.5 \%$ at adulthood (Fig. $1 \mathrm{i}$ and Supplementary Table 1). We then examined the dynamics of $\mathrm{Zeb}^{+}$basal cells during prostate regression and regeneration. As shown in Fig. 1g, $1 \mathrm{j}$ and Supplementary Table 1 , the proportion of the $\mathrm{Zeb} 1^{+} \mathrm{CK} 5^{+}$population became augmented in regressed prostates, and then decreased to the intact prostate level after regeneration, suggesting that they are more resistant to castration.

\section{Zeb1 $^{+}$basal cells are enriched for multipotent prostate basal stem cells}

To test the role of $\mathrm{Zeb}^{+}$basal cells in prostate development, we performed a prostate organoid-forming assay in vitro using flow cytometry sorted Zeb1 ${ }^{+}$and Zeb1' basal cells (Fig. 2a, b). While few and small organoids were produced from Zeb1 ${ }^{-}$basal cells, significantly larger and more organoids were generated from Zeb1 ${ }^{+}$basal cells (Fig. 2c, 2e and Supplementary Table 1). Immunostaining analysis of frozen sections of organoids formed from sorted Zeb1 ${ }^{+}$basal cells showed generation of both basal (CK5, CK14 or p63 positive) and luminal (CK8 or AR positive) cells (Fig. 2d). Moreover, Zeb1 ${ }^{+}$basal cells possessed a serial organoid forming capacity, indicating a self-renewing characteristic (Fig. 2e and Supplementary Table 1). We then evaluated the potential of $\mathrm{Zeb}^{+}$basal cells to generate prostates in vivo, a gold standard to assess the stem cell phenotype ${ }^{8,32,33}$. Using an in vivo kidney capsule transplantation assay, we grafted 1000 $\mathrm{Zeb1}^{+}$or $\mathrm{Zeb}^{-}$mouse prostate basal cells in combination with rat embryonic urogenital sinus 
mesenchymal (UGM) stromal cells under the renal capsule of host athymic nude mice. Compared to opaque and small grafts derived from Zeb1 ${ }^{-}$basal cells, Zeb1 ${ }^{+}$basal cells generated semitranslucent and large grafts (Supplementary Fig. 1a-c). Histological analysis indicated that Zeb1 ${ }^{+}$ grafts underwent ductal morphogenesis with differentiation of basal $\left(\mathrm{CK} 5^{+}\right)$, luminal $\left(\mathrm{CK}^{+}\right)$and rare neuroendocrine $\left(\mathrm{Syp}^{+}\right)$cell lineages (Supplementary Fig. 1d, 1f). Pbsn expression further confirmed differentiation of functional secretory luminal cells (Supplementary Fig. 1f). In addition, $\mathrm{Zeb}^{+}$grafts expressed the mouse $\beta$-integrin, which validated their mouse origin and indicated that they were not produced from the contamination of rat epithelial cells from the preparation of rat UGM cells (Supplementary Fig. 1f). We further verified that the generated prostate tissues were originated from $\mathrm{Zeb1}^{+}$donor cell grafts by positive staining of an MHC class I haplotype $\mathrm{H}-2 \mathrm{k}^{\mathrm{b}}$ protein that is specifically expressed in C57BL/6 but not host athymic mouse cells (Supplementary Fig. 1f). These data suggested that $\mathrm{Zeb}^{+}$basal cells are multipotent prostate stem cells and possessed the capacity of generating functional prostates.

\section{Single Zeb1 ${ }^{+}$basal cell can generate functional prostate in vivo}

We then wondered whether a single $\mathrm{Zeb}^{+}$basal cell would generate a prostate in vivo. Single viable Zeb1 ${ }^{+}$or Zeb1 ${ }^{-}$basal cell was FACS sorted into individual well of a 96-well plate, mixed with rat UGM stromal cells and transplanted into the renal capsules of nude mice. Each well was examined under the microscope to confirm the presence of a tdTomato ${ }^{+}$or tdTomato $^{-}$single cell (Fig. 2f). Fifteen prostates with well-differentiated prostate epithelial tubules containing all three basal, luminal and neuroendocrine cell lineages were generated from 36 single Zeb1 ${ }^{+}$basal cell transplants (Fig. 2g-q). In contrast, only 3 prostates were produced from 33 single Zeb1 basal cell xenografts (Fig. 2g-q). Furthermore, Zeb $1^{+}$Epcam $^{+}$cells harvested from the first generation of 
xenografted prostate tissues were able to form functional prostates in the secondary and tertiary sub-renal capsule transplantations (Supplementary Fig. 2). Collectively, these data provided additional direct evidences that $\mathrm{Zeb}^{+}$basal cells represented a multipotent prostate stem cell population.

In vivo lineage tracing supports that $\mathrm{Zeb1}^{+}$basal cells give rise to basal, luminal and neuroendocrine progeny

To further determine the role of $\mathrm{Zeb}^{+}$basal cells in prostate development in vivo, we generated a Zeb1-CreERT2 mouse line and crossed it with Rosa-CAG-LSL-tdTomato mice to trace Zeb1 ${ }^{+}$ basal cells during the postnatal prostate development (Fig. 3a, b). Tamoxifen administration to postnatal day 3 Zeb1-CreERT2/tdTomato mice induced expression of tdTomato in $10 \%$ of Zeb1 ${ }^{+}$ cells (Fig. 3c). Consistent with our immunostaining results and findings from the Zeb1-tdTomato knockin mouse model (Fig. 1), about $0.33 \%$ of $\mathrm{CK}^{+}$basal cells were labeled by tdTomato but none of the $\mathrm{CK}^{+}$luminal cells nor $\mathrm{Syp}^{+}$neuroendocrine cells were labeled at 2 days after induction (Fig. 3d-f, 3i and Supplementary Table 1). Interestingly, 12 days after the tamoxifen administration, we found clusters of $\mathrm{CK}^{+} \mathrm{CK} 8{ }^{-}$tdTomato $^{+}$cells located in the outer basal cell layer as well as $\mathrm{CK}^{-}$ $\mathrm{CK}^{+}$tdTomato $^{+}$cells in the inner luminal compartment (Fig. 3g, 3i and Supplementary Table 1). Quantitative analysis exhibited that labeled basal cells expanded more than 16 folds and tdTomato expressing luminal cells increased from zero to around 4\% in 12 days. Meanwhile, we also observed that about $3 \%$ of $\mathrm{Syp}^{+}$neuroendocrine cells were marked with tdTomato at 12 days post induction (Fig. 3h). Those results further substantiated the notion that Zeb1 ${ }^{+}$basal cells were able to generate basal, luminal and neuroendocrine cell lineages. 


\section{Zeb1 is required for development of prostatic basal cells}

We then asked whether Zeb1 was functionally required for normal prostate development. To that end, we first utilized the clustered regularly interspaced short palindromic repeats (CRISPR)/CRISPR-associated protein 9 (Cas9) system to achieve Zeb1 gene knockout in prostate organoids in vitro (Fig. 4a). Efficient Zebl deletion in mouse primary prostate epithelial cells was validated by immunoblotting (Fig. 4b). Zeb1 sgRNA transfected prostate organoids contains CK8 ${ }^{+}$ luminal cells, however, Zeb1 knockout organoids displayed a marked decrease of p63 expressing basal cells compared to organoids formed from mock-transfected prostate epithelial cells (Fig. 4c, $\mathrm{d}$ and Supplementary Table 1). We next investigated the role of Zeb1 in prostate development in vivo. Due to the fact that Zeb1 knockout mice died shortly after birth ${ }^{34}$, UGS from E16 Zeb1/embryos were dissected and transplanted beneath the renal capsule of male athymic mice to allow us to assess prostate epithelial development with Zeb1 deletion (Fig. 4e) . Although Zeb1 ${ }^{-/-}$UGS grafts developed into prostate and contains prostatic ductal structures, Zeb1 null grafts were smaller (Fig. 4e, f). Detailed histological examination revealed that approximately $50 \%$ of the $\mathrm{Zeb}^{-/-}$UGS derived prostate epithelia virtually displayed all luminal cells that are positive of CK8 and AR without basal cells. In the other prostate epithelia, basal cell numbers were greatly reduced (Fig. 4f-h and Supplementary Table 1). Further immunofluorescent co-staining with Zeb1 and p63 antibodies affirmed Zeb1 deletion and marked decrease of basal cells in Zeb1 ${ }^{-/-}$UGS grafts (Fig. 4g, h and Supplementary Table 1). These findings were further supported with additional staining neuroendocrine cells could still be found in Zeb1 $1^{-/-}$UGS derived prostate epithelium (Fig. 4g). Collectively, our in vitro and in vivo data highlight an indispensable role for Zeb1 in prostate basal cell development. 


\section{Unbiased single cell transcriptomic analysis of prostate basal cells identifies a unique Zeb1+ cell subset which expresses both epithelial and mesenchymal gene signatures}

To provide additional evidence for the existence of a Zeb1 ${ }^{+}$basal population, we performed unbiased single cell transcriptomic analysis of prostate basal cells. Using the 10x Genomics single cell system followed by Illumina sequencing, prostate basal cells (lineage-Sca ${ }^{+} \mathrm{CD} 49 \mathrm{f}^{\mathrm{hi}}$ ) ${ }^{15}$ were purified from mouse prostates via FACS for single-cell RNA sequencing. Sorted basal cells were confirmed by q-RT-PCR and immunostaining for expression of basal cell markers (Supplementary Fig. 3a-c). The flow chart for single-cell RNA sequencing data analysis was included in Supplementary Fig. 3d. To visualize cellular heterogeneity, 9833 single-cell transcriptome data were subjected to unsupervised Seurat clustering and projected onto two dimensions by tdistributed stochastic neighbor embedding (t-SNE). After removal of low-quality and contaminated non-epithelial cells (Supplementary Fig. 3e, f and 4), this led to a total of 9 prostate basal cell clusters (Cluster 1-9 or termed as C1-C9) (Fig. 5a). Importantly, we further found that while all other basal cell clusters displayed high levels of epithelial genes, the cell cluster C7, expressed both epithelial markers Epcam, Cldn1, Ocln and stromal cell markers including Vim, Fn1, and Acta2, stood out uniquely among all the cell clusters (Fig. 5a, b and Supplementary Table 2). Detailed analysis of highly variable genes in C7 showed that Zebl was exclusively expressed in C7 (Fig. 5a, b and Supplementary Table 2). Other EMT transcriptional factors including Zeb2,

Snai1, Prrx1 and Prrx2 were also highly transcribed in C7 (Fig. 5a, b and Supplementary Table 2). This EMT-like cell cluster can also be identified through the Monocle cell clustering method (Supplementary Fig. 5a, b). Interestingly, when we used statistical and computational frameworks to delineate the differentiation states of the 9 cell clusters, both monocle and diffusion map scripts 
indicated that C7 was at one of the tips in the lineage tree (Supplementary Fig. 5). Collectively, the unbiased examination of single-cell gene expression profiles of prostate basal cells further substantiates a unique Zeb1 expressing basal cell subpopulation with the mesenchymal gene signature.

\section{Wnt signaling pathway is enriched in Zeb1 ${ }^{+}$basal cells}

As an effort to uncover signaling pathways that are enriched in the cell cluster $\mathrm{C}$, we applied gene ontology (GO) enrichment analysis to the single-cell RNA-seq data. Coincidently, we found that the Wnt signaling pathway, which we have reported recently to promote self-renewal of prostate cancer stem cells ${ }^{35}$, was one of the top hits preferentially presented in the cluster C7 (Fig. 5c and Supplementary Table 3). The Wnt signaling pathway was demonstrated previously to play an essential positive role in inducing EMT ${ }^{36}$. Our RT-PCR experiments confirmed a significant upregulation of key Wnt signaling components, EMT transcriptional factor and mesenchymal markers, and downregulation of epithelial makers in Zeb1 ${ }^{+}$basal cells (Fig. 5d). It was recently reported that Procr, a Wnt pathway target, marks a novel multipotent mammary stem cell population ${ }^{37}$. Consistently, we also found a moderate (1.5 fold) but significant upregulation of Procr in $\mathrm{Zeb}^{+}$prostate basal cells (Fig. 5d). Further flow cytometry examination revealed that $\mathrm{Zeb}^{+}$cells represented only a small subpopulation (8\%) of Procr expressing prostate epithelial cells (Supplementary Fig. 6), suggesting a more enrichment of PBSCs in Zeb1 ${ }^{+}$than Procr prostate epithelial cells. Furthermore, examination of prostate sections from APC ${ }^{\text {min }}$ mice with the aberrantly activated Wnt pathway revealed a significant increase of Zeb1 ${ }^{+}$basal cells (Fig. 5e, $\mathrm{f}$ and Supplementary Table 1), suggesting a positive role of the Wnt signaling in the expansion of Zeb1 ${ }^{+}$basal cells. 


\section{Zeb1-expressing cells can be detected in prostate epithelia of $\mathrm{Hi}$-Myc mice and human prostate samples}

We next asked the influence of oncogenic transformation on the Zeb1 expression profile in the prostate epithelium. For that purpose, we crossed the Zeb1/tdTomato mice with the $\mathrm{Hi}-\mathrm{Myc}$ prostate cancer mouse model. Immunofluorescent co-staining of Zeb1 or tdTomato together with basal or luminal cell markers revealed a 2-fold increase of Zeb1+ basal cells in Zeb1/tdTomato; HiMyc prostates $(1.25 \%$ versus $0.73 \%$ in control mice at 3 -month old, or $1.71 \%$ versus $0.71 \%$ in control mice at 5-month old) (Fig. 6a, b and Supplementary Table 1). Of note, we detected a small number of $\mathrm{Zeb}^{+} \mathrm{CK} 8^{+}$luminal cells in HiMyc mice, which stood in contrast to the exclusive expression of Zeb1 in the basal cell compartment from wild type prostates (Fig. 6a, b and Supplementary Table 1). Similarly, positive immunostaining of Zeb1 protein could be found in a small percent of $\mathrm{CK} 14^{+}$basal cells in human prostate tissues (non-benign prostate hyperplasia (non-BPH) $(n=3)$ and BPH specimens $(n=7))$ (Fig. 6c, d and Supplementary Table 1). During the examination of many sections of human prostate cancer samples, we infrequently found cancer tissues (Gleason score: $3+3$ or $3+4, n=4$ ) with remaining $\mathrm{CK} 14^{+}$basal cells, in which Zeb1 positive staining could be found (Fig. 6c, d and Supplementary Table 1). Intriguingly, Zeb1 ${ }^{+} \mathrm{CK} 8^{+}$luminal cells were barely detected in non-BPH but could be observed occasionally in BPH specimens and more frequently in prostate cancer specimens (Fig. 6e). Together, Zeb1 expressing epithelial cells can be detected in both basal and luminal layers of prostates from $H i-M y c$ mice and in human prostate samples. 


\section{Discussion}

The prostate basal cell compartment is suggested to contain stem/progenitor cells ${ }^{6,8,15,21}$, but the stem cell subpopulation within prostate basal cells and its transcriptional profile remain largely unknown. We here discover the exclusive existence of Zeb1-positive prostate epithelial cells in a small population of basal compartment but not in the luminal layer. Zeb $1^{+}$basal cells are more frequently found in the stem cell enriched urethra-proximal region. Importantly, utilizing functional methods and in vivo lineage tracing, we prove that the Zeb1 $1^{+}$prostate basal cells can self-renew and possess multipotency to generate all three prostatic epithelial cell lineages in vivo. Furthermore, in vitro and in vivo genetic ablation experiments showed a requirement of Zeb1 in basal cell differentiation. The unique Zeb1 expressing prostate basal cell cluster with mesenchymal cell features was further supported by single-cell RNA sequencing data. Our study underscores the importance of Zeb1 as a single marker and an essential regulator for PBSCs.

EMT has been demonstrated to be utilized by tumor initiating cells to acquire stem cell attributes ${ }^{23}$. We find here that Zeb1 marks a population of PBSCs in prostates with mesenchymal features and higher expression levels of EMT inducers such as Snai1, Zeb2, Prrx1 and Prrx2. Support for our data comes from recent studies in mammary glands that multipotent Procr $^{+}$ mammary stem cells display EMT characteristics and that EMT is required for the tissuereconstitution activity of mammary basal cells ${ }^{37-40}$. Together, those observations demonstrate that like what was shown for tumor initiating cells, EMT is also closely associated with stemness in normal prostate and mammary stem cells. However, there is also tissue specific difference between stem cells in prostate versus mammary tissues. Firstly, we find that although almost all Zeb1 ${ }^{+}$ prostate basal cells express Procr, Procr is present in a much larger population of prostate epithelial cells containing both basal and luminal cells (Supplementary Fig. 6 and data not shown), suggesting 
that Zeb1 appears to be a better maker for basal stem cells in prostates than Procr. Secondly, Zeb1 and Snai 1 are expressed in prostate basal cells but not in mammary epithelial cells ${ }^{38-40}$, indicating distinct EMT inducing transcriptional factors or EMT programs are involved in respective stem cells.

The serial organoid forming and transplantation assays together with lineage tracing experiments of Zeb1 $1^{+}$basal cells in the current study demonstrate that Zeb1 ${ }^{+}$basal cells can selfrenew and give rise to basal, luminal and neuroendocrine cell lineages. Loss of function experiments performed on prostate organoids and UGS transplants show that Zeb1 knockout results in a remarkably decrease in the basal cell compartment, but luminal and neuroendocrine cell differentiation are largely unaffected. Consistently, previous studies on renal capsule transplants from p63 ${ }^{-/-}$UGS exhibit development of luminal and neuroendocrine cells independent of basal cells $^{41}$. In addition, lineage tracing experiments on CK14, CK8, CK5 and NKX3.1 cells support the existence of self-sustained luminal cell progenitors $5,6,9,10$. Therefore, both multipotent $\mathrm{Zeb1}^{+}$basal stem cells and stem/progenitor cells from the luminal compartment contribute to prostate development.

Intriguingly, we find that different from the exclusive basal expression of Zeb1 in wild-type prostate, $\mathrm{Zeb1}^{+}$epithelial cells can be detected in the luminal compartment in Hi-myc mice or in human prostate hyperplastic or tumor samples. There are two possible explanations for the appearance of Zeb1 ${ }^{+}$luminal cells: It could be derived from transformed Zeb1 ${ }^{+}$basal cells; or alternatively, the transformed luminal cells may acquire Zeb1 expression. Clarification of these two possibilities needs further investigations. Nevertheless, the appearance of Zeb1 ${ }^{+}$luminal cells at early stages of prostate tumorigenesis is of special interest, because most clinical prostate cancers display a dominant luminal phenotype by loss of basal cell phenotypes, and Zeb1 has been 
well shown in the acquisition of cancer stem cell properties ${ }^{28,42}$. This Zeb $1^{+}$luminal cell population might represent prostate tumor initiating cells, which can be also related to two different cellular origins for prostate cancers: basal versus luminal cells.

To our knowledge, this work is the first report to decipher the heterogeneity within the prostate basal epithelium using unbiased single-cell transcriptomics. Importantly, along with the single-cell RNA-seq data, our immunocytochemical staining, functional experiments, and cell lineage tracing analysis together support that Zeb1 marks a multipotent prostate basal stem cells and Zeb1 itself is required for prostate basal cell development. The identification of the novel Zeb $1^{+}$prostate stem cell, its transcriptome profile and its expression pattern during prostate tumorigenesis could have important implications for our understanding of tissue development and regeneration and for identification of potential cellular origin for cancer.

\section{Acknowledgements}

The study is supported by funds to by funds to W-Q Gao from the National Key R\&D Program of China (2017YFA0102900), the National Natural Science Foundation of China (NSFC, 81630073 and 81872406), the Science and Technology Commission of Shanghai Municipality (16JC1405700), the Education Commission of Shanghai Municipality (for the High Peak IV subject on Stem Cells and Translational Medicine Research) and the KC Wong foundation, and by funds to H.H. Zhu from the NSFC (81772743), Shanghai Municipal Education CommissionTong University (Excellent Youth Scholar Initiation Grant 16XJ11003). 


\section{Author contributions}

H.H.Z. and W.Q.G. conceived the study; X.W. performed the experiments. H. X. and D. W. conducted bioinformatic analyses; Z. J. and J. W. assisted in data interpretation; C. C. and Y. S. helped in cell culture experiments; H.Z. helped microscopic imaging; X. L. performed mouse genotyping and breeding; Y. S. helped organoid culture; Y. H. assisted in animal experiments. L.F., B.D., and W.X. collected human prostate samples and assisted in histological analysis of human sample; H.H.Z., W.Q.G. and X.W. interpreted the data and wrote the manuscript.

Declaration of Interests: The authors declare no competing interests. 


\section{Reference}

1. Shen, M.M. \& Abate-Shen, C. Molecular genetics of prostate cancer: new prospects for old challenges. Genes Dev 24, 1967-2000 (2010).

2. Isaacs, J.T. Control of cell proliferation and cell death in the normal and neoplastic prostate: A stem cell model., Vol. II 85-94. (National Institute of Health, Report No. 87-2881, 1987).

3. Wang, J. et al. Symmetrical and asymmetrical division analysis provides evidence for a hierarchy of prostate epithelial cell lineages. Nature communications 5, 4758 (2014).

4. Wang, Z.A. et al. Lineage analysis of basal epithelial cells reveals their unexpected plasticity and supports a cell-of-origin model for prostate cancer heterogeneity. Nat Cell Biol 15, 274-283 (2013).

5. Choi, N., Zhang, B., Zhang, L., Ittmann, M. \& Xin, L. Adult murine prostate basal and luminal cells are selfsustained lineages that can both serve as targets for prostate cancer initiation. Cancer Cell 21, 253-265 (2012).

6. Ousset, M. et al. Multipotent and unipotent progenitors contribute to prostate postnatal development. Nat Cell Biol 14, 1131-1138 (2012).

7. Karthaus, W.R. et al. Identification of multipotent luminal progenitor cells in human prostate organoid cultures. Cell 159, 163-175 (2014).

8. Leong, K.G., Wang, B.E., Johnson, L. \& Gao, W.Q. Generation of a prostate from a single adult stem cell. Nature 456, 804-808 (2008).

9. Wang, X. et al. A luminal epithelial stem cell that is a cell of origin for prostate cancer. Nature 461, 495-500 (2009).

10. Chua, C.W. et al. Single luminal epithelial progenitors can generate prostate organoids in culture. Nat Cell Biol 16, 951-961, 951-954 (2014).

11. Xin, L., Lawson, D.A. \& Witte, O.N. The Sca-1 cell surface marker enriches for a prostate-regenerating cell subpopulation that can initiate prostate tumorigenesis. Proceedings of the National Academy of Sciences of the United States of America 102, 6942-6947 (2005).

12. Kwon, O.J., Zhang, L. \& Xin, L. Stem Cell Antigen-1 Identifies a Distinct Androgen-Independent Murine Prostatic Luminal Cell Lineage with Bipotent Potential. Stem Cells 34, 191-202 (2016).

13. Yoo, Y.A. et al. Bmil marks distinct castration-resistant luminal progenitor cells competent for prostate regeneration and tumour initiation. Nat Commun 7, 12943 (2016). 
14. Zhang, D. et al. Histone 2B-GFP Label-Retaining Prostate Luminal Cells Possess Progenitor Cell Properties and Are Intrinsically Resistant to Castration. Stem Cell Reports 10, 228-242 (2018).

15. Lawson, D.A., Xin, L., Lukacs, R.U., Cheng, D. \& Witte, O.N. Isolation and functional characterization of murine prostate stem cells. Proceedings of the National Academy of Sciences of the United States of America 104, 181-186 (2007).

16. Goldstein, A.S. et al. Trop2 identifies a subpopulation of murine and human prostate basal cells with stem cell characteristics. Proceedings of the National Academy of Sciences of the United States of America 105, 20882-20887 (2008).

17. Kwon, O.J. \& Xin, L. Prostate epithelial stem and progenitor cells. Am J Clin Exp Urol 2, 209-218 (2014).

18. Goldstein, A.S., Huang, J., Guo, C., Garraway, I.P. \& Witte, O.N. Identification of a cell of origin for human prostate cancer. Science 329, 568-571 (2010).

19. Xin, L., Lukacs, R.U., Lawson, D.A., Cheng, D. \& Witte, O.N. Self-renewal and multilineage differentiation in vitro from murine prostate stem cells. Stem Cells 25, 2760-2769 (2007).

20. Stoyanova, T. et al. Prostate cancer originating in basal cells progresses to adenocarcinoma propagated by luminal-like cells. Proceedings of the National Academy of Sciences of the United States of America 110, 20111-20116 (2013).

21. English, H.F., Santen, R.J. \& Isaacs, J.T. Response of glandular versus basal rat ventral prostatic epithelial cells to androgen withdrawal and replacement. Prostate 11, 229-242 (1987).

22. Aigner, K. et al. The transcription factor ZEB1 (deltaEF1) promotes tumour cell dedifferentiation by repressing master regulators of epithelial polarity. Oncogene 26, 6979-6988 (2007).

23. Shibue, T. \& Weinberg, R.A. EMT, CSCs, and drug resistance: the mechanistic link and clinical implications. Nat Rev Clin Oncol 14, 611-629 (2017).

24. Mayer, C. et al. Developmental diversification of cortical inhibitory interneurons. Nature (2018).

25. Zheng, C. et al. Landscape of Infiltrating T Cells in Liver Cancer Revealed by Single-Cell Sequencing. Cell 169, 1342-1356 e1316 (2017).

26. Nieto, M.A., Huang, R.Y., Jackson, R.A. \& Thiery, J.P. Emt: 2016. Cell 166, 21-45 (2016).

27. Zhang, L., Zhao, W., Valdez, J.M., Creighton, C.J. \& Xin, L. Low-density Taqman miRNA array reveals miRNAs differentially expressed in prostatic stem cells and luminal cells. The Prostate 70, 297-304 (2010). 
28. Mani, S.A. et al. The epithelial-mesenchymal transition generates cells with properties of stem cells. Cell 133, 704-715 (2008).

29. Li, P. et al. Zeb1 promotes androgen independence of prostate cancer via induction of stem cell-like properties. Experimental biology and medicine 239, 813-822 (2014).

30. Salm, S.N. et al. TGF-\{beta\} maintains dormancy of prostatic stem cells in the proximal region of ducts. $J$ Cell Biol 170, 81-90 (2005).

31. Tsujimura, A. et al. Proximal location of mouse prostate epithelial stem cells: a model of prostatic homeostasis. J Cell Biol 157, 1257-1265 (2002).

32. Donjacour, A.A. \& Cunha, G.R. Assessment of prostatic protein secretion in tissue recombinants made of urogenital sinus mesenchyme and urothelium from normal or androgen-insensitive mice. Endocrinology 132, 2342-2350 (1993).

33. Xin, L., Ide, H., Kim, Y., Dubey, P. \& Witte, O.N. In vivo regeneration of murine prostate from dissociated cell populations of postnatal epithelia and urogenital sinus mesenchyme. Proceedings of the National Academy of Sciences of the United States of America 100 Suppl 1, 11896-11903 (2003).

34. Takagi, T., Moribe, H., Kondoh, H. \& Higashi, Y. DeltaEF1, a zinc finger and homeodomain transcription factor, is required for skeleton patterning in multiple lineages. Development 125, 21 (1998).

35. Zhang, K. et al. WNT/beta-Catenin Directs Self-Renewal Symmetric Cell Division of hTERT(high) Prostate Cancer Stem Cells. Cancer Res 77, 2534-2547 (2017).

36. Scheel, C. et al. Paracrine and autocrine signals induce and maintain mesenchymal and stem cell states in the breast. Cell 145, 926-940 (2011).

37. Wang, D. et al. Identification of multipotent mammary stem cells by protein C receptor expression. Nature 517, $81-84$ (2015).

38. Ye, X. et al. Distinct EMT programs control normal mammary stem cells and tumour-initiating cells. Nature 525, 256-260 (2015).

39. Guen, V.J. et al. EMT programs promote basal mammary stem cell and tumor-initiating cell stemness by inducing primary ciliogenesis and Hedgehog signaling. Proceedings of the National Academy of Sciences of the United States of America 114, E10532-E10539 (2017). 
40. Guo, W. et al. Slug and Sox9 cooperatively determine the mammary stem cell state. Cell 148, 1015-1028 (2012).

41. Kurita, T., Medina, R.T., Mills, A.A. \& Cunha, G.R. Role of p63 and basal cells in the prostate. Development 131, 4955-4964 (2004).

42. Li, P., Yang, R. \& Gao, W.Q. Contributions of epithelial-mesenchymal transition and cancer stem cells to the development of castration resistance of prostate cancer. Molecular cancer 13, 55 (2014).

43. Lukacs, R.U., Goldstein, A.S., Lawson, D.A., Cheng, D. \& Witte, O.N. Isolation, cultivation and characterization of adult murine prostate stem cells. Nat Protoc 5, 702-713 (2010). 
Figures and Figure Legends

Fig. 1
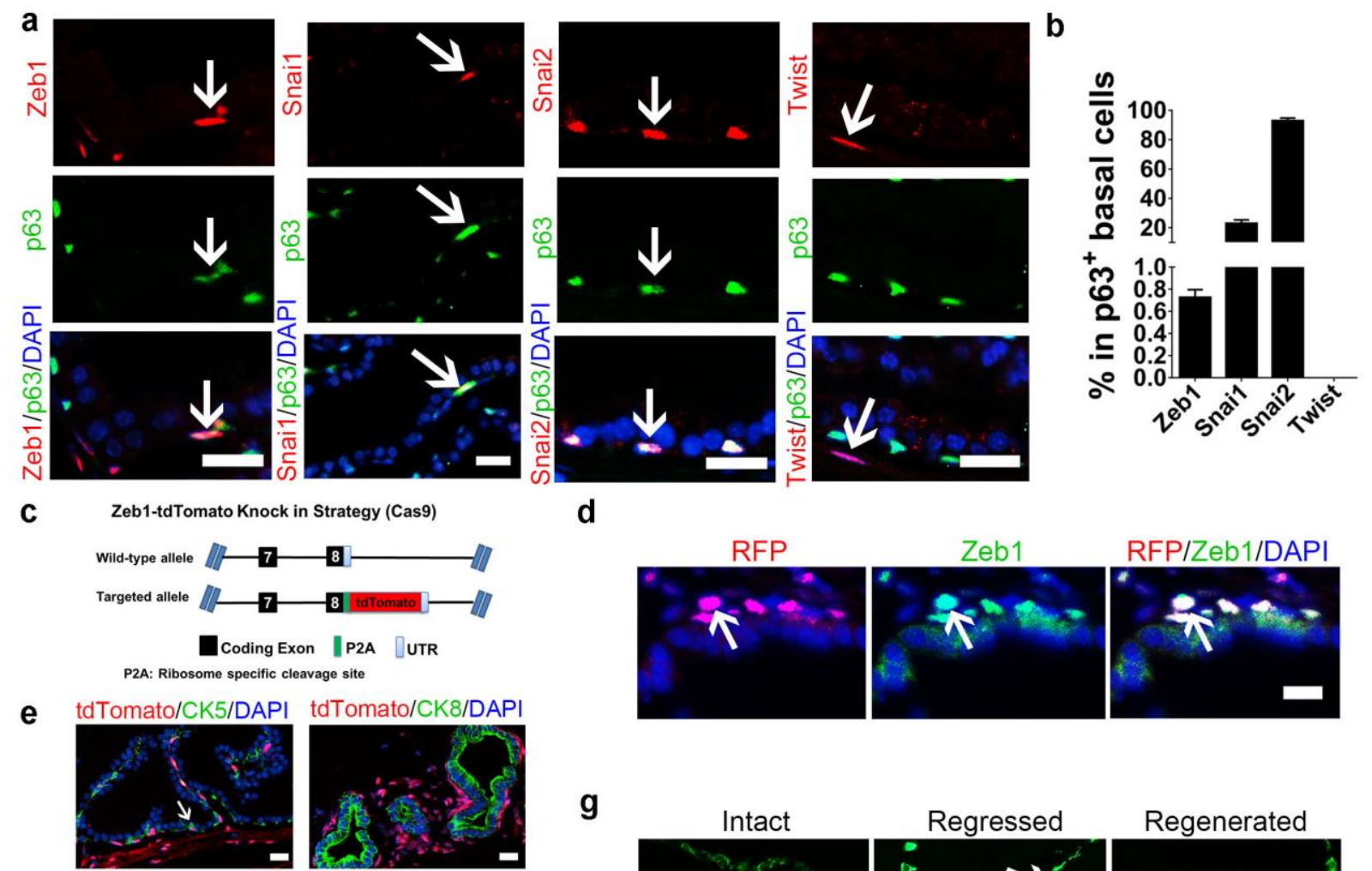

d
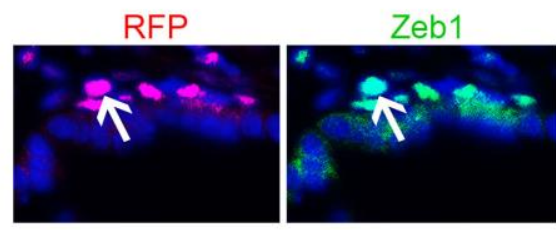

RFP/Zeb1/DAPI

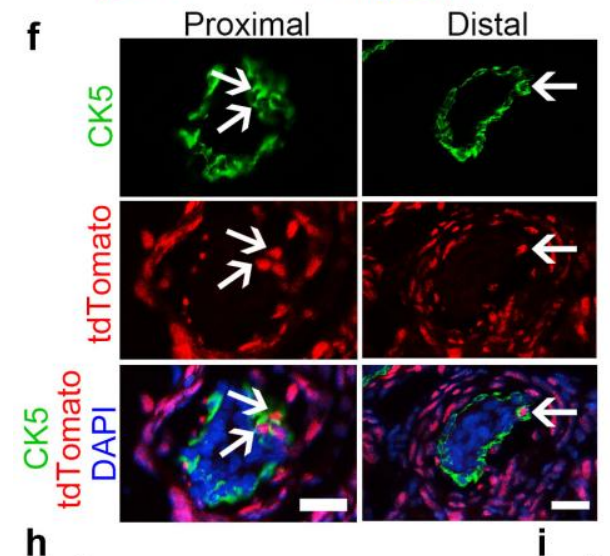

g
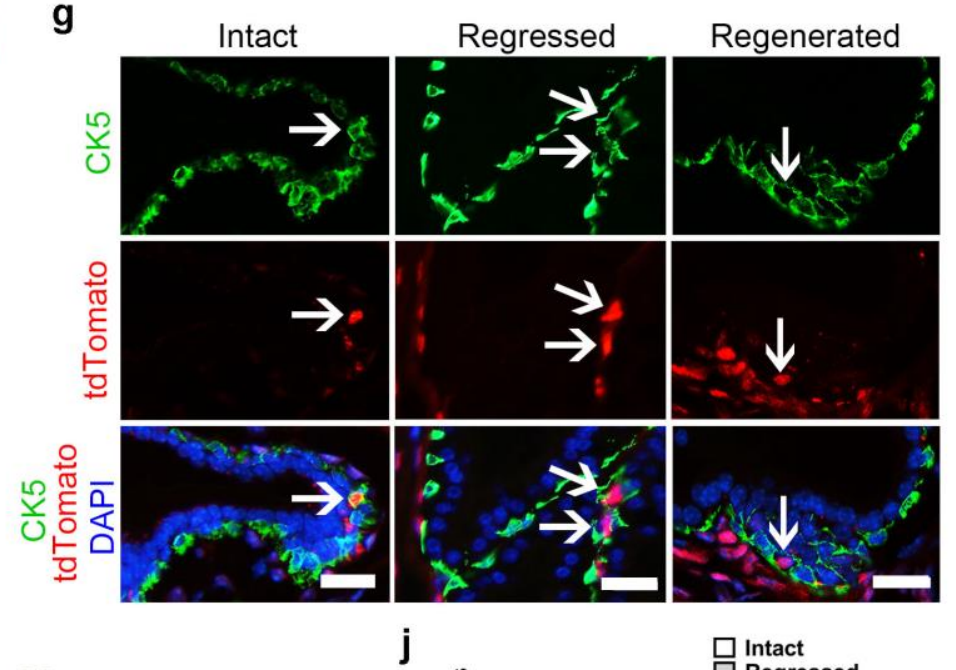

h
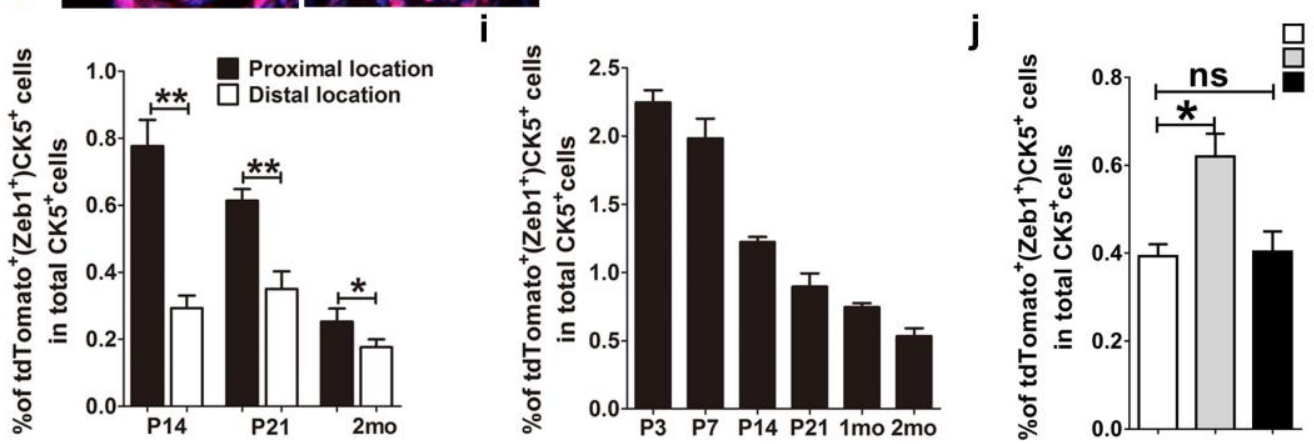

Figure 1 
Fig. 1| Mouse prostates contain a population of Zeb1+ basal cells that are more frequently detected in the urethra-proximal region.

a, Co-immunostaining for Zeb1, Snai1, Snai2 (Slug), or Twist1/2 together with p63 on frozen sections of p21 wild-type mouse prostates.

b, Quantification of the ratio of $\mathrm{Zeb}^{+}{ }^{+}$, Snai $1^{+}, \mathrm{Snai}^{+}$or Twist $1 / 2^{+}$cells in $\mathrm{p} 63^{+}$prostatic basal epithelial cells.

c, Illustration of the strategy to generate the Zeb1-tdTomato reporter mouse model.

d, Zeb1 and tdTomato co-staining on prostate sections from Zeb1-tdTomato reporter mouse confirms that the td-Tomato labeling faithfully indicated the endogenous expression of Zeb1.

e, Immunostaining of tdTomato and CK5 or CK8 on prostate frozen sections of 3-week-old wild type mice showing tdTomato expression is only found in prostate basal cells but not luminal cells. (Scale bars $=20 \mu \mathrm{m})$

f, The tdTomato $(\mathrm{Zeb} 1)^{+}$prostate basal cells are more frequently found in the urethra-proximal region compared to the distal region.

g, Section imaging of intact, regressed (21 days after castration) and regenerated (14 days after androgen replacement) prostate sections from Zeb1-tdTomato reporter mice.

$\mathbf{h}$, Quantification of tdTomato $(\mathrm{Zeb} 1)^{+}$prostate basal cells in urethra-proximal and distal regions at indicated developmental stages.

i, Quantification of tdTomato $(\mathrm{Zeb} 1)^{+}$prostate basal cells at indicated time points of prostate development.

j, Quantification of tdTomato $(\mathrm{Zeb} 1)^{+}$prostate basal cells at indicated time points of prostate regeneration.

(In this figure, at least 20 fields per section of 3 sections each mouse prepared from 3 mouse 
bioRxiv preprint doi: https://doi.org/10.1101/601872; this version posted April 8, 2019. The copyright holder for this preprint (which was not certified by peer review) is the author/funder. All rights reserved. No reuse allowed without permission.

prostates were analyzed. Data are analyzed by Student's t-test and are presented as mean + s.e.m. All scale bars $=20 \mu \mathrm{m}$.) 
Fig. 2

a

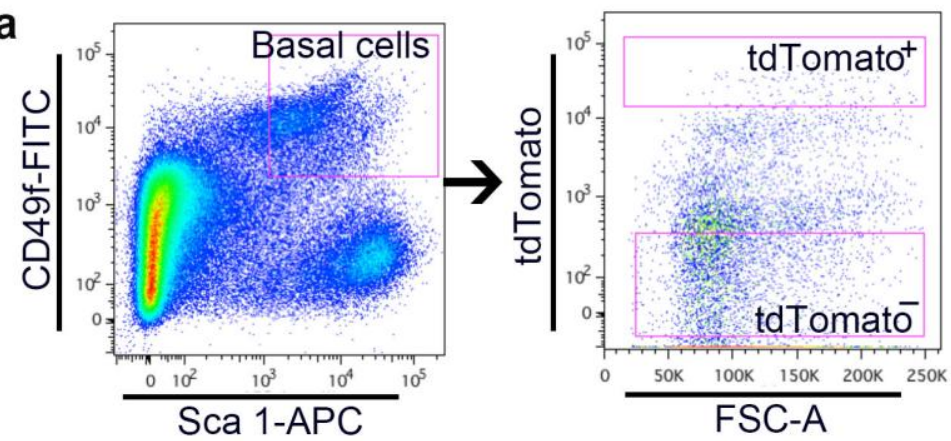

C
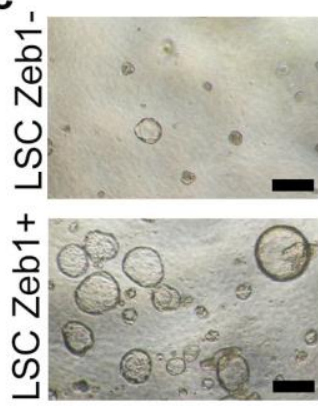

f
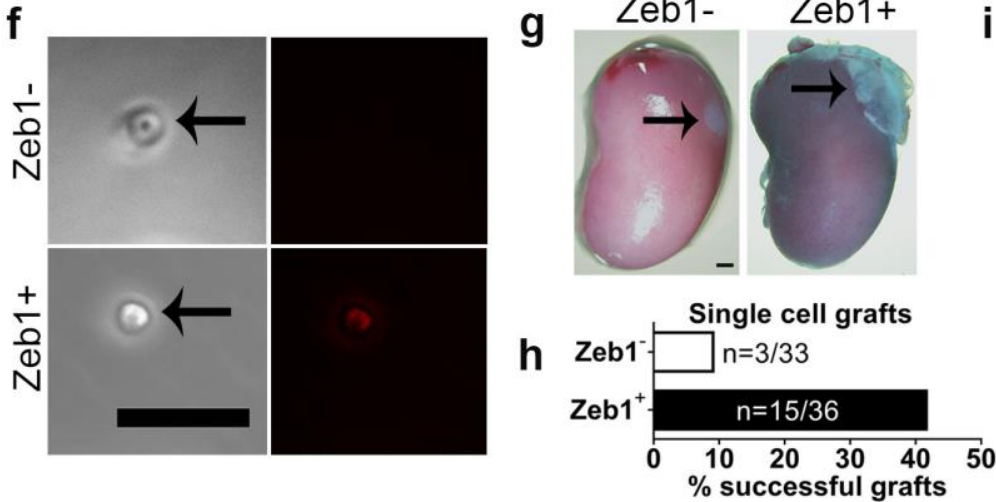

b

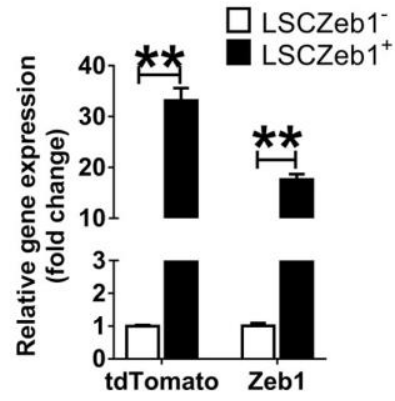

e

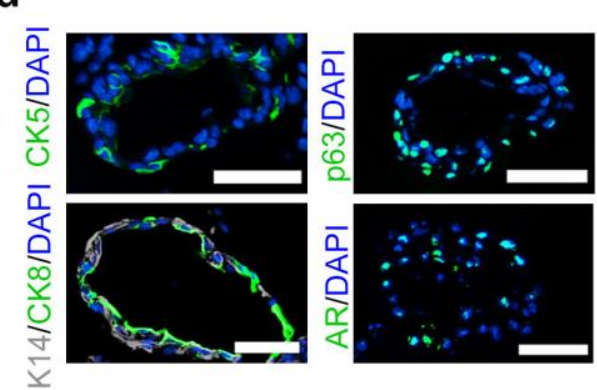

g

Zeb1- Zeb1+ i

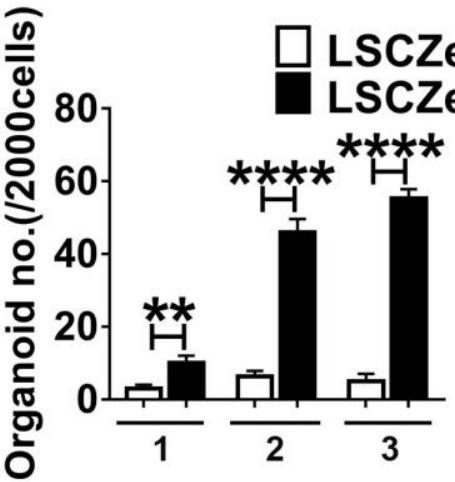

RFP/p63/DAPI
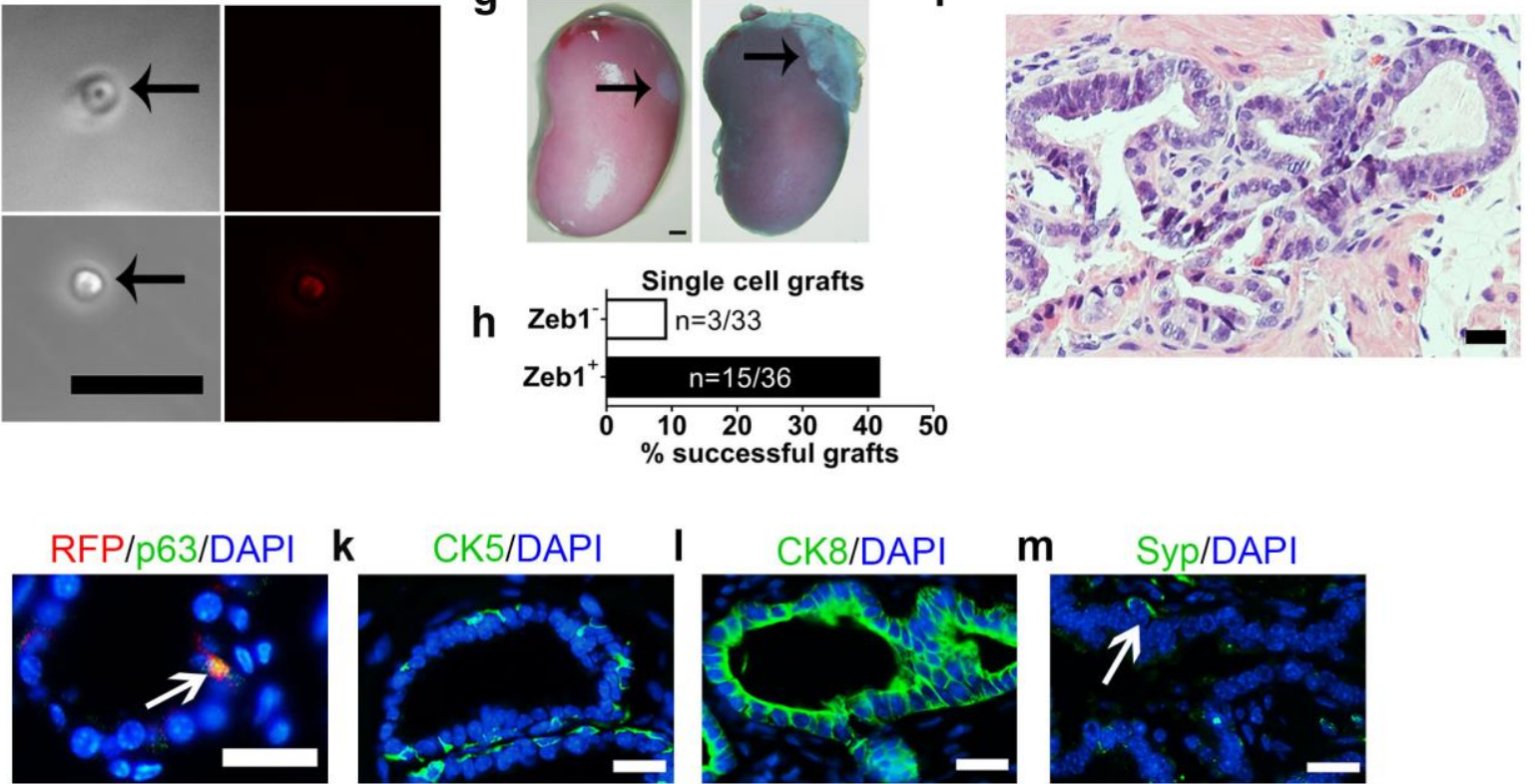

n
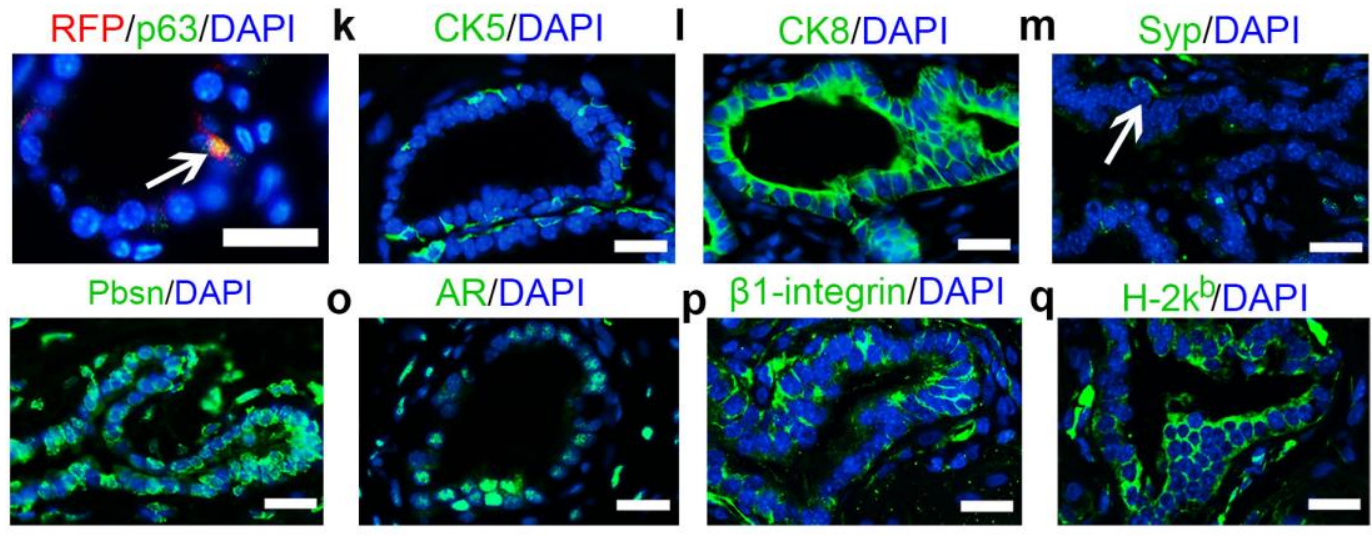

Figure 2 
Fig. 2| Zeb1+ basal cells are enriched for prostate stem cells.

a, b, FACS and qRT-PCR quantification of Zeb1expression in Lineage Sca- $^{+}$CD $49 \mathrm{f}^{\mathrm{hi}}$ tdTomato $^{+}$ and Lineage-Sca- $1^{+} \mathrm{CD} 49 \mathrm{f}^{\text {hi }}$ tdTomato ${ }^{-}$prostate cells. RNA expression levels were normalized to Lineage-Sca- $1^{+} \mathrm{CD} 49 \mathrm{f}^{\text {hi }}$ tdTomato ${ }^{-}$prostate basal cells. $(\mathrm{n}=5)$

c-e, In vitro serial organoid-forming assay showing more and larger organoids were produced from $\mathrm{Zeb}^{+}$basal cells compared to Zeb1 ${ }^{-}$basal cells. (Scale bars $=200 \mu \mathrm{m}$ for bright-field images and $50 \mu \mathrm{m}$ for the immunofluorescent images. The number 1,2,3 stands for the first, secondary and tertiary passaging.)

f, Phase contrast and fluorescent images of the single viable Lineage-Sca- $1^{+} \mathrm{CD} 49 \mathrm{f}^{\text {hi }} \mathrm{Zeb} 1^{+}$or Lineage $\mathrm{Sca}-1^{+} \mathrm{CD} 49 \mathrm{f}^{\text {hi }} \mathrm{Zeb1} 1^{-}$prostate cell used for single cell renal capsule transplantation experiments in vivo.

g, Prostate tissue generated from single Lineage $-\mathrm{Sca}-1^{+} \mathrm{CD} 49 \mathrm{f}^{\text {hi }} \mathrm{Zeb} 1^{+}$prostate cell at 2 months after renal capsule implantation.

$\mathbf{h}$, Quantification of prostate tissue generation incidence from single cell transplants.

i, H\&E staining of single cell implants showing differentiated prostate epithelial tubules.

j-o, Staining of RFP, p63 (j, green), CK5 (k), CK8 (l), Synaptosphysin (Syp) (m), Pbsn (n), and Androgen receptor (AR) (o) on sections of single cell implants. (The white arrow points to a Syp $^{+}$cell. Scale bars $\left.=20 \mu \mathrm{m}.\right)$

$\mathbf{p}, \mathbf{q}$, Staining of mouse-specific $\beta 1$-integrin (p) and C57BL/6 donor-specific $\mathrm{H}-2 \mathrm{k}^{\mathrm{b}}$ (q) on sections of single cell implants. (Scale bars=20 $\mu \mathrm{m}$.)

(data are analyzed by Student's t-test and are presented as mean + s.e.m.) 
Fig. 3

a

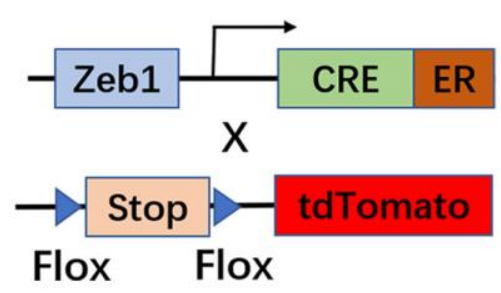

b

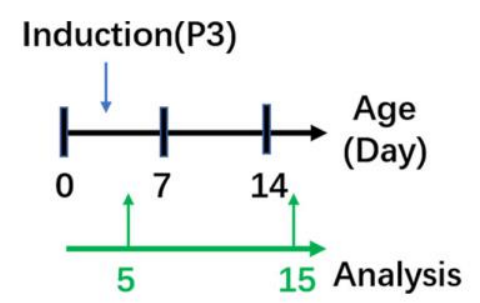

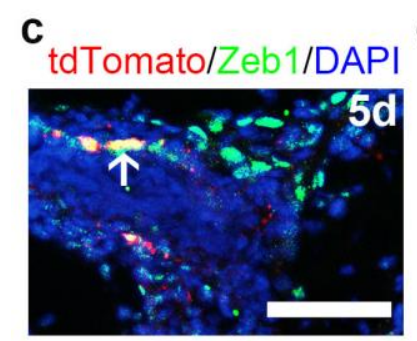

d tdTomato/CK5/DAPI
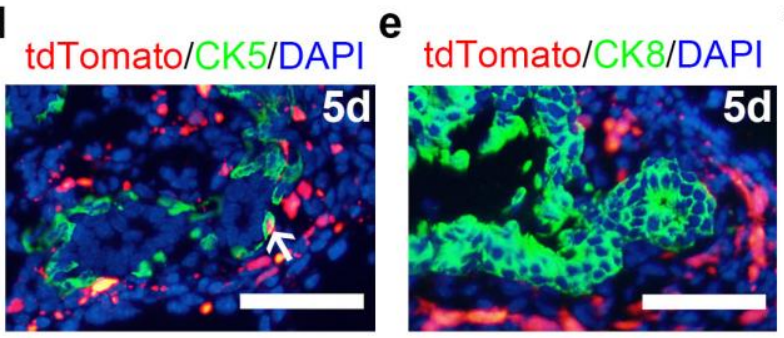

g

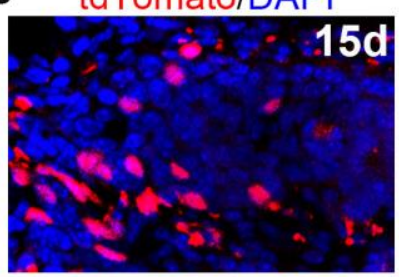

CK5/DAPI

CK8/DAPI
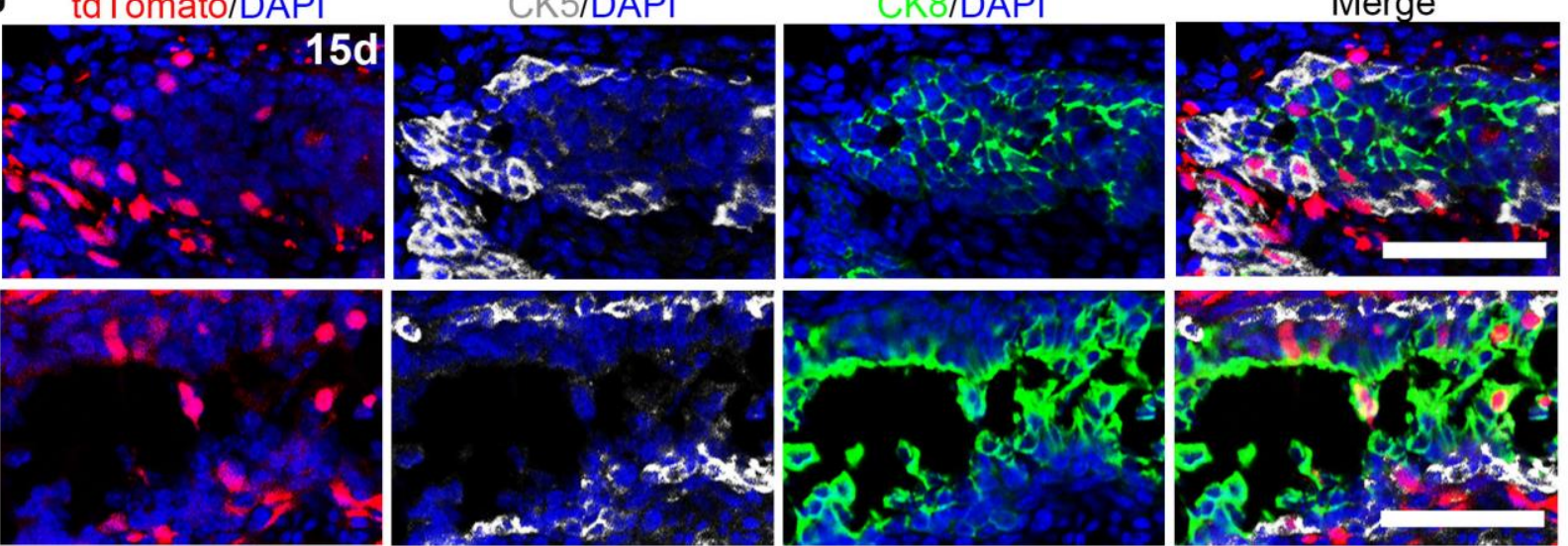

$\mathbf{h}$

tdTomato/Syp/DAPI
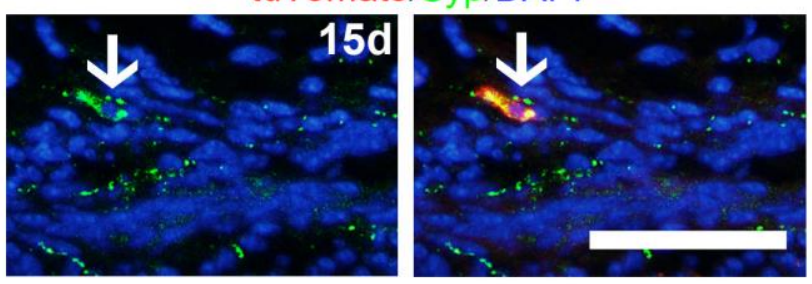
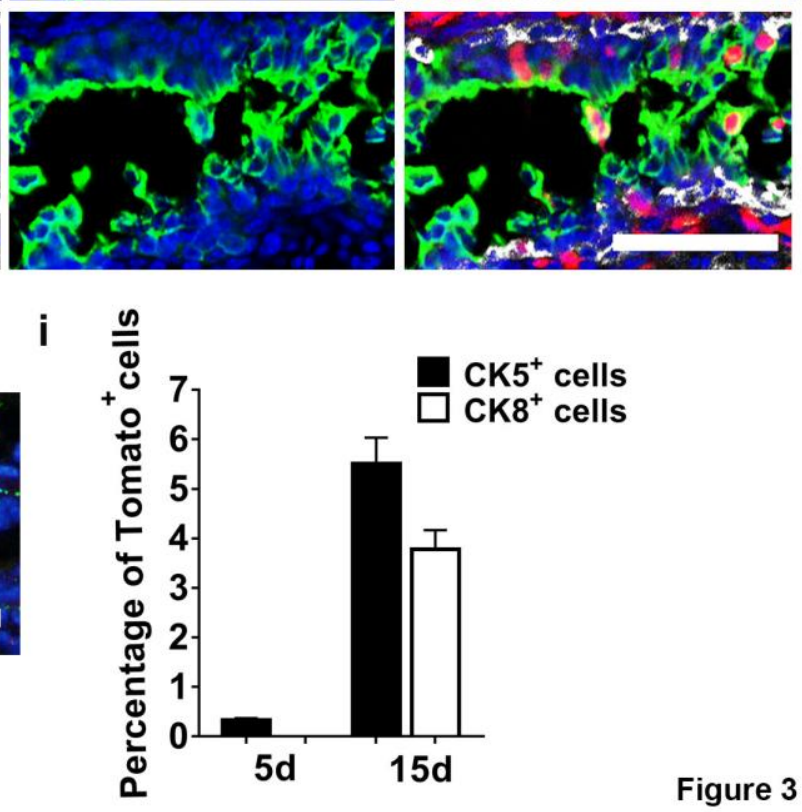

Figure 3 
Fig. 3| Lineage tracing supports that Zeb1 ${ }^{+}$basal cells give rise to basal, luminal and neuroendocrine cells

a, Strategy to target tdTomato expression in Zeb1 expressing basal cells in vivo.

b, Illustration of protocols to trace the fate of $\mathrm{Zeb} 1^{+}$basal cells during prostate postnatal development.

c, Zeb1 and tdTomato co-staining of prostate sections from Zeb1-CreERT2/tdTomato mice shows that tdTomato marks Zeb1 expressing cells.

d-f, tdTomato expression is found in $\mathrm{CK}^{+}$basal cells, but not in $\mathrm{CK} 8^{+}$luminal cells and $\mathrm{Syp}^{+}$ neuroendocrine cells from Zeb1-CreERT2/tdTomato mouse prostates at 2 days after tamoxifen administration.

g, Clusters of $\mathrm{CK}^{+} \mathrm{CK}^{-}{ }^{-} \mathrm{TT}^{-} \mathrm{mato}^{+}$cells can be detected in the outer basal cell layer and $\mathrm{CK}^{-}$ $\mathrm{CK}^{+}{ }^{+} \mathrm{tdomato}^{+}$cells can be found in the inner luminal compartment at 12 days after tamoxifen induction.

h, $\operatorname{Syp}^{+}$neuroendocrine cells are marked with tdTomato at 12 days post induction.

i, Percentage of tdTomato ${ }^{+}$cells in $\mathrm{CK}^{+}$and $\mathrm{CK}^{+}$cells at 2 days and 12 days after tamoxifen administration to postnatal day 3 mice.

(In this figure, at least 20 sections each mouse prepared from 3 mouse prostates were analyzed. At least 60 fields for each immunostaining experiment were collected for analysis. Data are analyzed by Student's t-test and are presented as mean + s.e.m. All scale bars $=50 \mu \mathrm{m}$.) 
Fig. 4

a

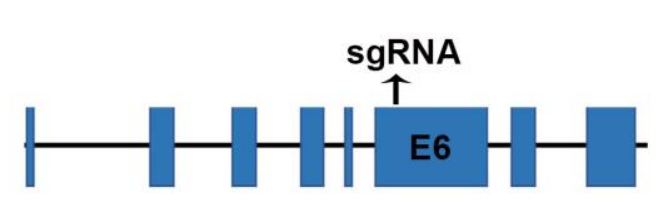

C

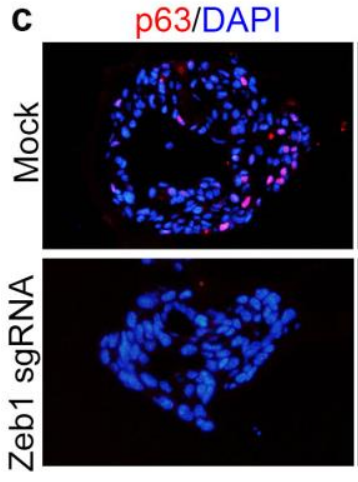

e

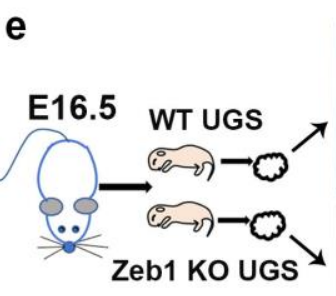

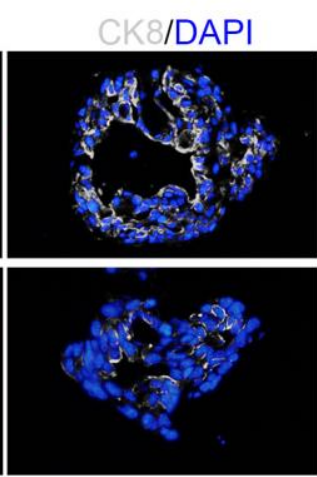
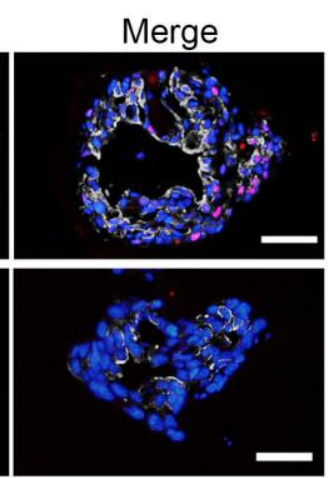

f

WT

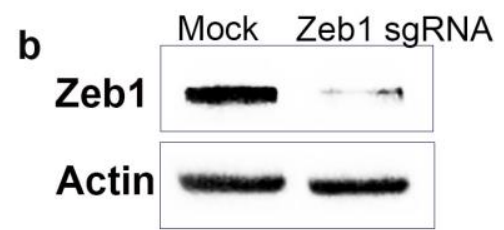

d

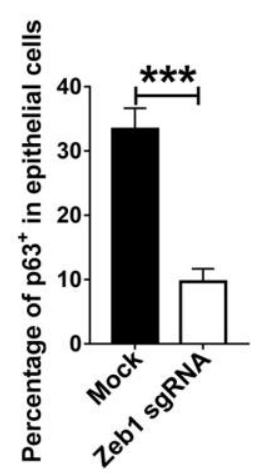

$\mathrm{KO}$
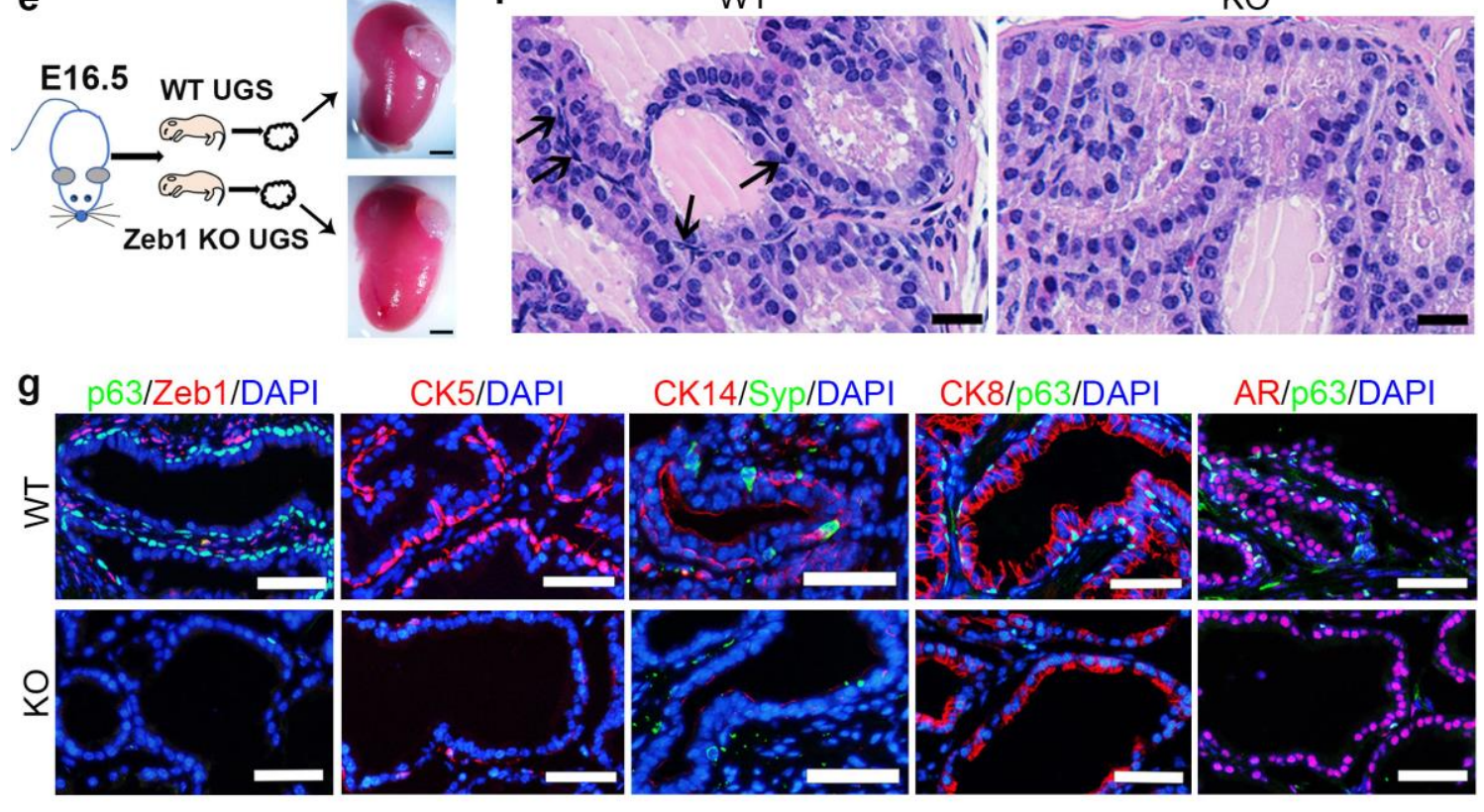

h

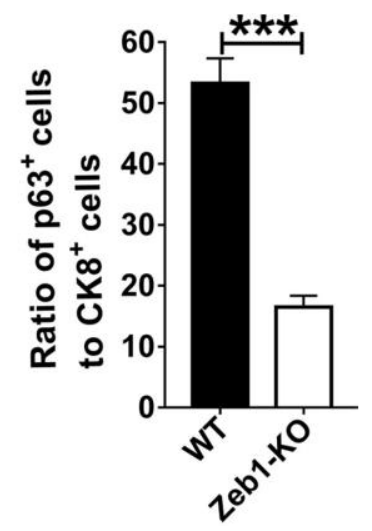

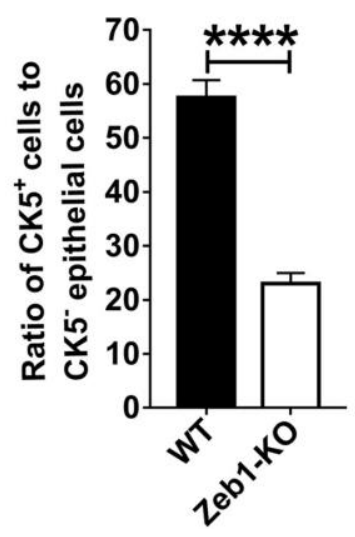

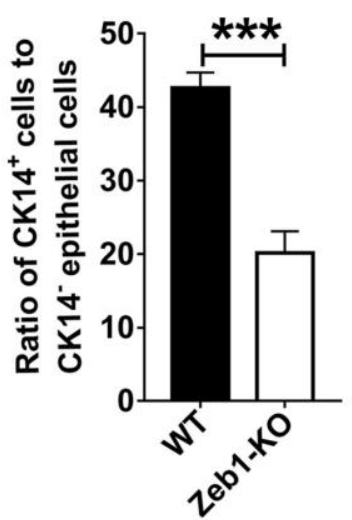

Figure 4 


\section{Fig. 4| Knockout of Zeb1 severely suppresses the basal cell development}

a, Illustration of sgRNA sequence for CRISPR/Cas9 system mediated Zeb1 knockout.

b, Immunoblotting confirms efficient Zeb1 ablation sgRNA transfected primary prostate epithelial cells.

c, d, Immunostaining of frozen sections from Zeb1 knockout organoids shows a severely impaired differentiation of basal cells. (Scale bars $=50 \mu \mathrm{m}$. Experiments were repeated for 3 times. Data are analyzed by Student's t-test and are presented as mean + s.e.m.)

e, UGS from E16 wild-type or Zeb1 ${ }^{-/-}$embryos are dissected and transplanted beneath the renal capsule of male athymic mice. Transplants are harvested at 20 days later. (Scale bars $=200 \mu \mathrm{m})$

f, H\&E staining of UGS implants showing differentiated prostate epithelial tubules but a remarkably decrease in basal cell number in Zeb1 $1^{-/-}$UGS grafts. (Scale bars $\left.=20 \mu \mathrm{m}\right)$

g, Staining of Zeb1, p63, CK5, CK14, Syp, CK8 and AR on sections of wild-type and Zeb1 knockout UGS implants. (Scale bars $=50 \mu \mathrm{m}$ )

h, Quantification of basal cell development in Zeb1 $1^{-/-}$UGS grafts. (At least 60 fields from 20 sections for each immunostaining experiment were analyzed. Data are analyzed by Student's ttest and are presented as mean + s.e.m.) 
Fig. 5

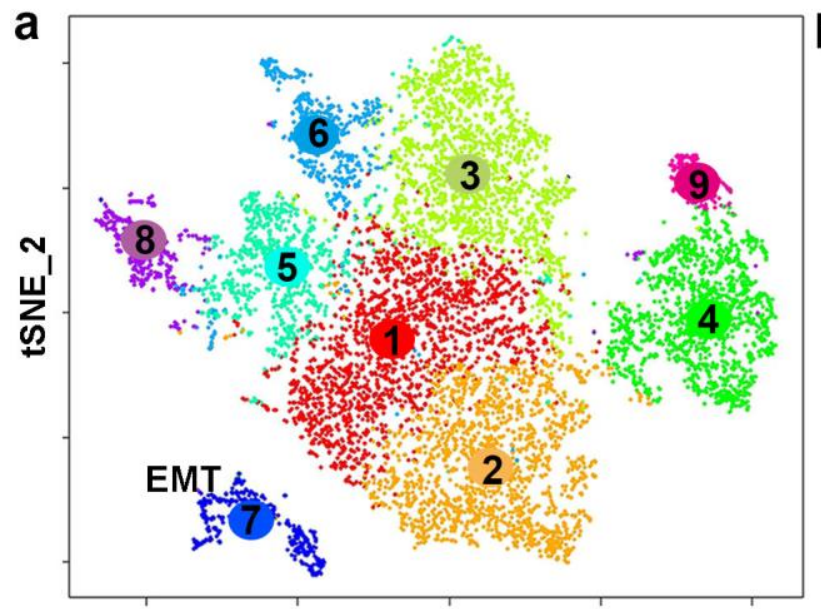

tSNE_1

C

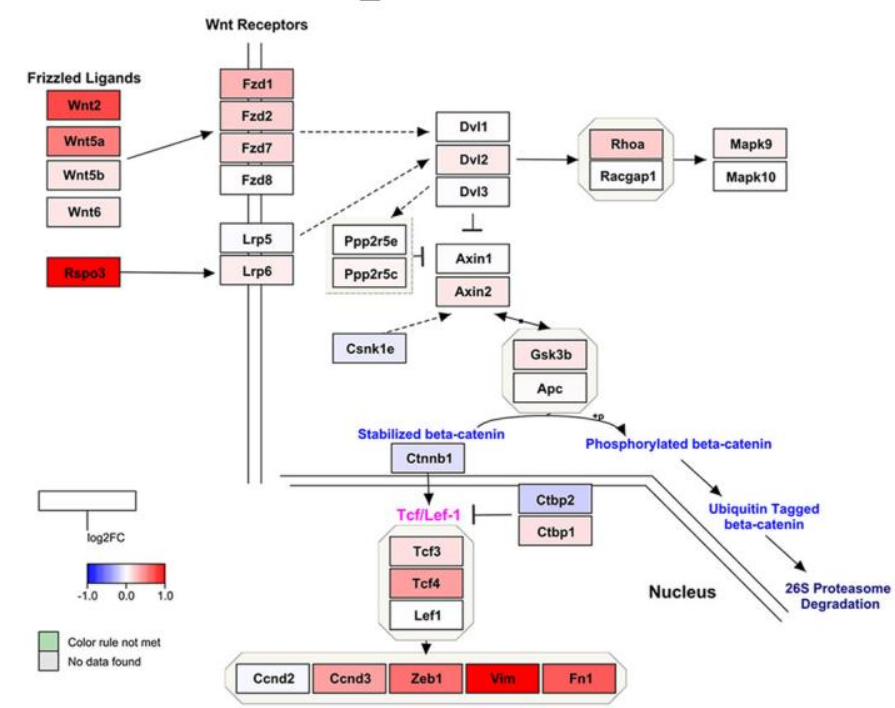

d

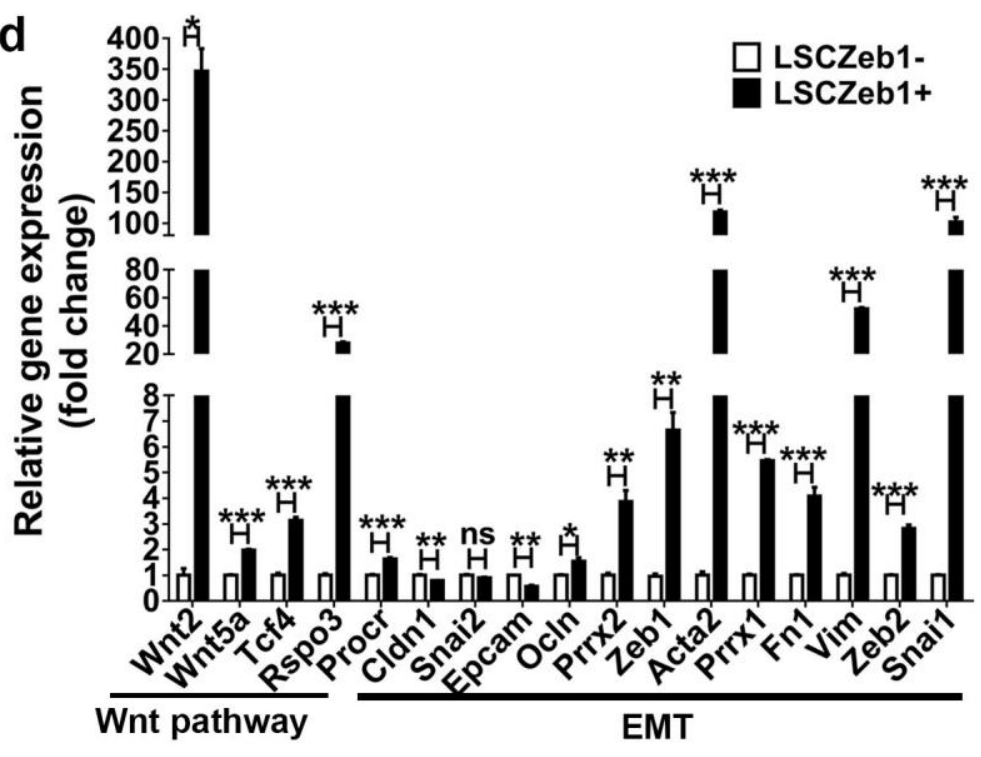

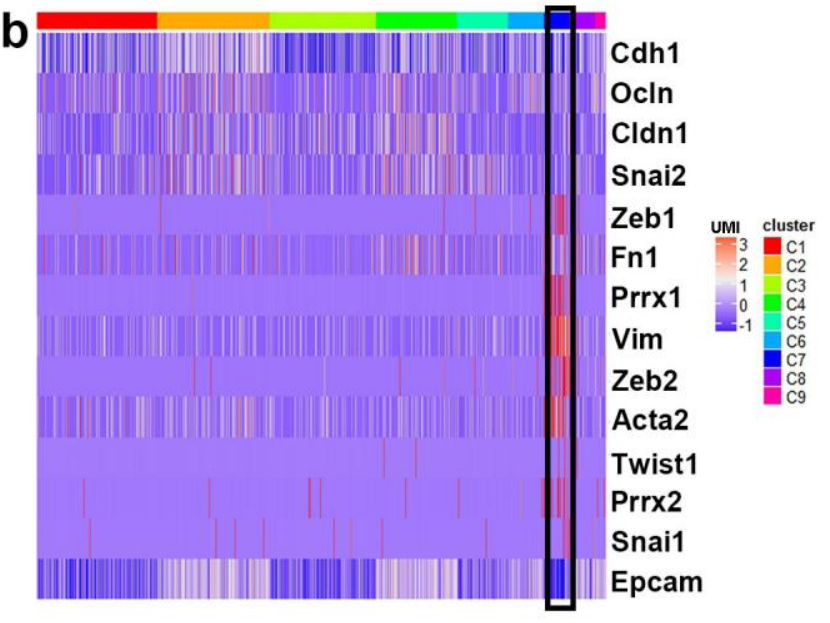

e

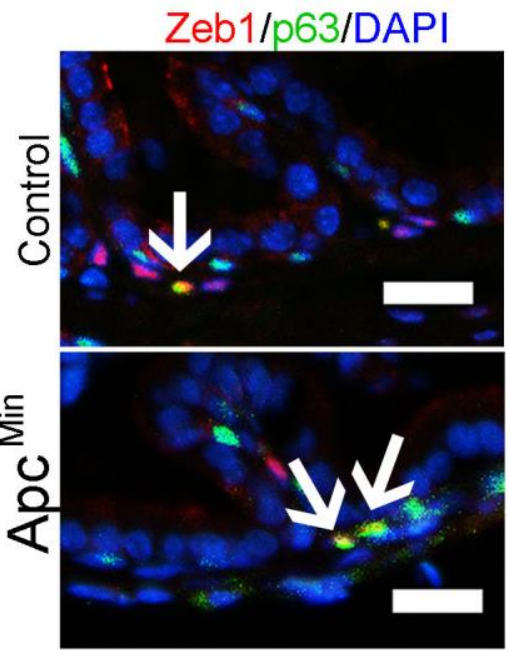

f

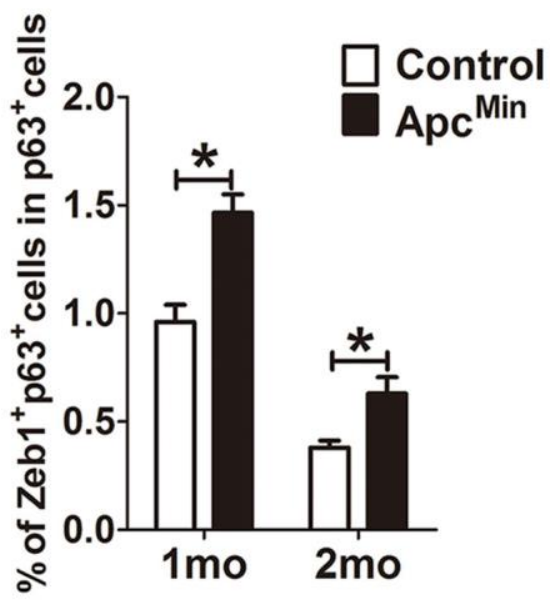

Figure 5 
Fig. 5| Single-cell RNA-seq data reveal that a Zeb1+ basal cell subset which shares gene transcriptional signatures with both epithelial and mesenchymal cells meanwhile preferentially expresses molecules in the Wnt signaling pathway and is expanded in APC ${ }^{\text {min }}$ mice.

a, A Seurat package and the first 12 principal components were applied to generate 9 different and stable clusters based on differential expressing genes among 9278 mouse prostate basal cells. C7 is marked with EMT due to its unique EMT expression profile.

b, A heatmap showing the scaled expression profile for EMT related genes. (The black-lined rectangle highlights cluster 7).

c, Color-coded expression levels of Wnt signaling pathway genes in the Zeb1 expressing Cluster 7 were presented as were obtained according to $\log 2$ fold change (log2FC) between cluster 7 and the rest prostate basal cell clusters. The pathway drawing was modulated from the schematic for the mouse Wnt pathway at the pathviso website.

d, qRT-PCR quantification of the mRNA expression of key components of the Wnt pathway and epithelial and stromal markers as well as EMT inducing transcriptional factors in Lineage-Sca$1^{+} \mathrm{CD} 49 \mathrm{f}^{\mathrm{hi}} \mathrm{Zeb} 1^{+}$and Lineage-Sca $-1^{+} \mathrm{CD} 49 \mathrm{f}^{\text {hi }} \mathrm{Zeb} 1^{-}$prostate cells. ( $\mathrm{n}=3$, data are analyzed by Student's t-test and are presented as mean + s.e.m.)

e, f, Co-immunostaining of Zeb1 and p63 on prostate sections reveals a significant increase of $\mathrm{Zeb}^{+}$basal cell number in $\mathrm{APC}^{\mathrm{min}}$ mice compared to control animals. (Scale bars $=20 \mu \mathrm{m}$ ) 
Fig 6

a

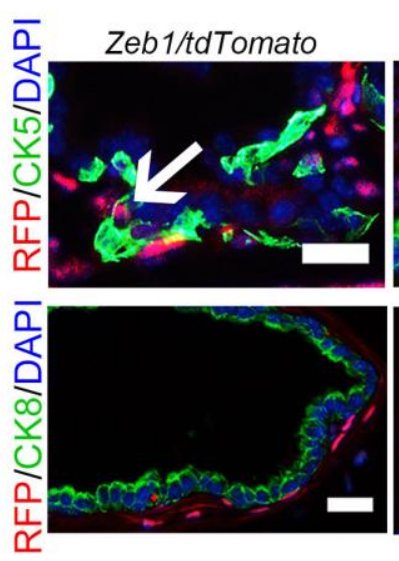

C
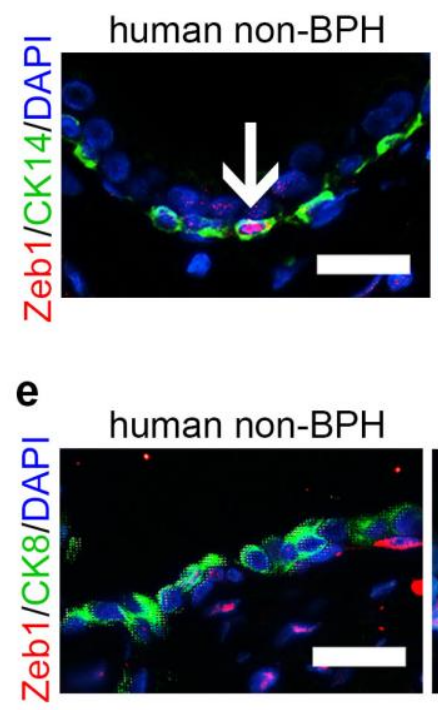

b

Zeb1/tdTomato; HiMyc
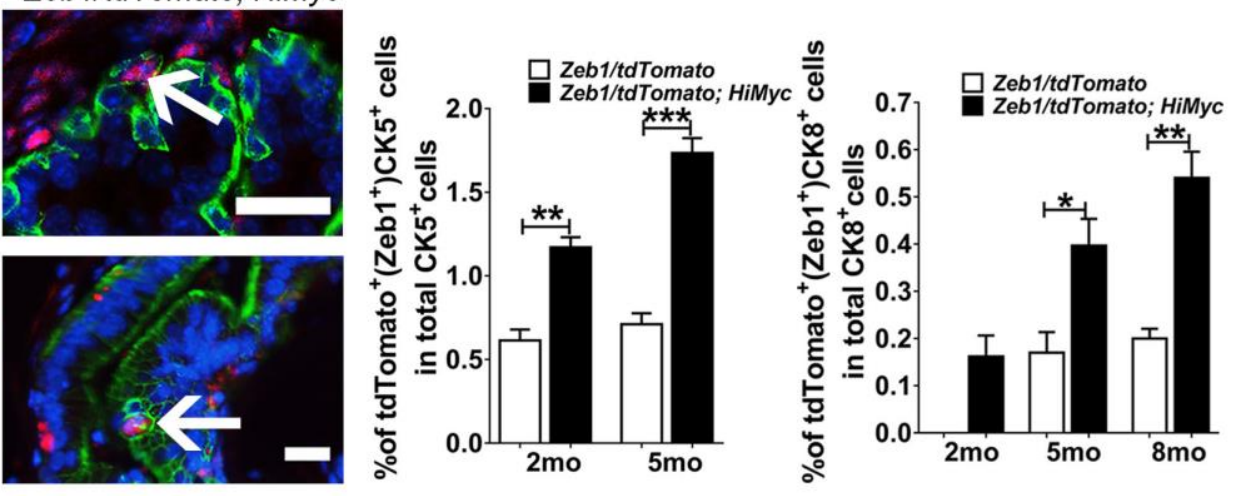

d

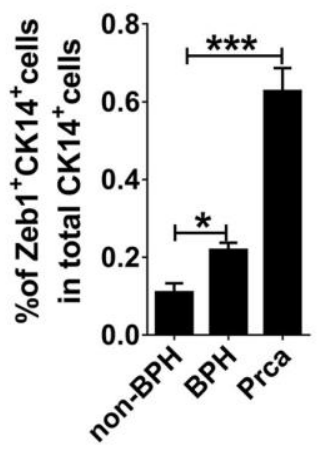

Figure 6 
Fig. 6| Zeb1+ epithelial cells are found in the basal layer of both mouse and human prostates and are detectable in both basal and luminal epithelium from mouse or human hyperplastic prostate tissues

a, Images of tdTomato and CK5 or CK8 staining on prostate sections from Zeb1/tdTomato or Zeb1/tdTomato; HiMyc mice.

b, Quantifications of tdTomato ${ }^{+}$basal or tdTomato ${ }^{+}$luminal cells in prostates from Zeb1/tdTomato or Zeb1/tdTomato; HiMyc mice. (In panel a-d, at least 20 fields per section of 3 sections each mouse prepared from 3 mouse prostates were analyzed. Data are analyzed by Student's t-test and are presented as mean + s.e.m. All scale bars $=20 \mu \mathrm{m}$.) cancer specimens.

e, Images of Zeb1 and CK8 co-immunostaining on sections from human non-BPH, BPH or prostate cancer specimens (In panel c-e, non-BPH $(n=3), B P H(n=7)$. For cancer samples, Gleason score $=3+3$ or $3+4, n=4$. Scale bars $=25 \mu \mathrm{m})$. 


\section{Methods}

\section{Animals}

Pregnant SD rats (E17) and athymic nu/nu male mice (7 weeks old) were purchased from Shanghai Slac laboratory animal company. Zeb1/tdTomato reporter mice were generated by the CRISPRCas9 method at Model Animal Research Center of Nanjing University. The sgRNA sequence we used is GAGGTTGGAGCTGCACAGCAGG. Exon 8 of Zeb1 followed by a P2A and tdTomato coding sequence, which was flanked by approximately $1.5 \mathrm{~kb}$ upstream and $1.5 \mathrm{~kb}$ downstream sequence of Zeb1 Exon 8, was cloned to a donor vector. Plasmid containing sgRNA and Cas9 expressing elements and the donor vector were injected to fertilized C57/B6 mouse eggs. F0 generation of mice were genotyped by sequencing and PCR. Positive F0 mice were used to backcross with C57/B6 mice to produce F1 generation of knockin mice. PCR Primers for Zeb1/tdTomato mouse genotyping were provided in Supplementary Table 4. Zeb1-CreERT2 mice were generated by a homologous recombination method at the Cyagen Biosciences Inc. The TAG stop codon of the last exon of Zeb1 was replaced with the "2A-CreERT2" cassette. Homology arms of the targeting vector were generated by PCR using BAC clone RP23-51G9 or RP23-207F18 from the C57BL/6J library as template. The targeting vector contained the Neo cassette flanked by SDA (self-deletion anchor) sites and DTA for negative selection. C57BL/6 ES cells was used for gene targeting. F0 generation of mice were validated by sequencing and PCR. PCR Primers for genotyping of Zeb1-CreERT2 mice were provided in Supplementary Table 4. Zebl knockout with deletion of exon 1 mice was purchased from RIKEN BioResource Research Center. The RosaCAG-LSL-tdTomato mice were purchase from the Jackson laboratory. Hi-Myc mice were introduced from the National Cancer Institute (NCI:01XF5). $A p c^{\text {min }}$ mice were introduced from the Nanjing Biomedical Research Institute of Nanjing University (T001457). All animal experiments 
were conducted according to the ethical regulations of Ren Ji Hospital. Animal experiment protocol were approved by the Ren Ji Hospital Laboratory Animal Use and Care Committee.

\section{Prostate single cell preparation}

Mouse prostates were harvested, minced, then digested with pre-warmed 1X collagenase/hyaluronidase solution for $3 \mathrm{hr}$ at $37^{\circ} \mathrm{C}$, washed with PBS buffer once and placed in $0.25 \%$ Trypsin/EDTA for 6 min at $37^{\circ} \mathrm{C}$. FBS supplemented with $4 \% \mathrm{FBS}$ was added to quench trypsin reaction followed by centrifuging at $350 \mathrm{~g}$ for $5 \mathrm{~min}$. Then, the cell pellet was resuspended with pre-warmed Dispase/DNase I solution to thoroughly dissociate cells. Single cell suspension was obtained from passing through a $40 \mu \mathrm{m}$ cell strainer.

\section{Flow cytometry}

Mouse prostate single cell suspension was blocked with the Fc blocker (CD16/32 antibody from eBioscience) for 40min at room temperature. Staining antibodies were diluted in $4 \%$ FBS buffer containing Y-27632 ROCK inhibitor and applied to the prostate single cell suspension for 40min at room temperature. Flow cytometry analysis or sorting was conducted using BD Accuri C6 or FACSAria III flow cytometer. Antibodies used in the study are listed in Supplementary Table 5. Propidium Iodide (PI, Invitrogen) was added to sample before sorting to gate viable cells.

\section{Single cell RNA-seq and data analysis}

Lineage-Sca- $1^{+} \mathrm{CD} 49 \mathrm{f}^{\text {hi }} \mathrm{PI}^{-}$prostate cells from postnatal day 14 mice were sorted and counted manually before processing to the single cell RNA-seq library preparation. Libraries were constructed following the instruction of the Chromium single cell 3' solution (10x Genomics). The 
libraries were sequenced on Illumina Hiseq X Ten platform. 10x Genomics workflow and Cell Ranger Single Cell Software was used to process raw sequencing data and to align reads to the mm10 mouse reference genome. Raw gene expression matrix produced by 10x Genomics workflow were first processed by the Seurat package. Cells with less than 5000 unique molecular identifiers (UMIs) or less than 1400 genes detected or more than 5\% UMI mapped to mitochondria genes were removed (Supplementary Fig. 3e, f). This led to 9833 high-quality single-cell RNA-seq data with an average gene detected to be around 2703 (Supplementary Fig. 3f). 1606 variable genes were selected based on their expression and dispersion (expression cutoff $=0.0125$ and dispersion cutoff=0.5). The first 12 principal components were used for clustering analysis (resolution=0.5) and t-SNE projection. This identified a total of 10 clusters (Supplementary Fig. 4a). Violin plots of epithelial, stromal, endothelial and immune cell associated genes for those 10 different cell clusters were presented in Supplementary Fig. 4b-e. Clusters which expressed clear markers of nonepithelial cells were removed in the following analyses. Cluster 5 was labeled as endothelial cells based on the expression of Eng, S1pr1 and Emcn (Supplementary Fig. 4d). Cluster 10 was labeled as immune cells based on the expression of Cd74 and Cd72 (Supplementary Fig. 4e). After the removal of above-mentioned non-epithelial cells, we re-ran the Seurat work flow to generate new cell clusters and t-SNE projections (the first 12 principal components were used). A total of 9 clusters were identified (Fig. 5a). We used the FindAllMarkers script in the Seurat package to identify genes that are enriched in a specific cluster (the specific cluster vs. the rest of clusters) with default settings. PCA matrix with the first 12 principal components and clusters from the second Seurat running were taken as input. The cluster representing putative stem cells (Zeb1 expressing cluster 7) was chosen as the root node. Totally, three branches were identified. Heatmaps were plotted by using ComplexHeatmap and ggplot2 package. Similar clusters and the structure of a 
common origin could also be inferred by using Monocle or Diffusionmap with standard parameters (Supplementary Fig. 5). Codes used are deposited to the website https://github.com/HelenHeZhu/StemCell.

\section{Human prostate clinical specimens}

Freshly dissected human prostate specimens (non-BPH, BPH and prostate cancer specimens) were obtained from the department of Urology at Ren Ji Hospital with informed consent from patients. All Human sample experiments were conducted according to the ethical regulations of Ren Ji Hospital. Human sample collection and handling protocols were approved by the Ren Ji Ethics committee.

\section{UGM stromal cell preparation}

The UGM isolation procedures has been described previously ${ }^{43}$. Briefly, E17 embryos from pregnant SD rats were sacrificed and urogenital sinuses were collected. The UGM was separated from the urogenital sinus epithelium and then digested with $1 \mathrm{X}$ collagenase/hyaluronidase solution on a shaker for $90 \mathrm{~min}$ at $37^{\circ} \mathrm{C}$. The UGM was washed with PBS once and then placed in $0.25 \%$ Trypsin/EDTA for $6 \mathrm{~min}$ at $37^{\circ} \mathrm{C}$. UGM single cell suspension was seeded to cell culture dishes and cultured in DMEM supplemented with 10\% fetal bovine serum (FBS), 2mM glutamine, $100 \mathrm{U} / \mathrm{ml}$ penicillin and $100 \mathrm{mg} / \mathrm{ml}$ streptomycin in vitro for at least 1 week.

\section{Serial isolation and renal capsule transplantation}

We used a previously described procedure to isolate the primary prostate epithelial cells and to perform renal capsule transplantation ${ }^{43}$. For the first renal capsule transplantation, single or 
indicated number of Lineage ${ }^{-S c a}-1^{+} \mathrm{CD} 49 \mathrm{f}^{\mathrm{hi}}$ tdTomato $^{+}$or Lineage $\mathrm{Sca}-\mathrm{H}^{+} \mathrm{CD} 49 \mathrm{f}^{\mathrm{hi}}$ tdTomato $^{-}$ prostate basal cell was sorted into each well of a 96-well plate via FACS and then examined under a light microscope. The viable sorted cell was mixed with purified rat UGM cells $(250,000$ cells per graft) in rat tail Collage Type I (4.42mg/ml, Corning, 354236). Next, the mixture was plated as a drop of $10 \mu \mathrm{l}$ into the center of a well of a 6 -well plate. The plate was placed in a $37^{\circ} \mathrm{C}$ cell culture incubator for at least $60 \mathrm{~min}$ to make sure that the Collage type I solidified. Then pre-warmed DMEM (containing 10\%FBS) medium was gently pipetted into each well. Each collagen graft was embedded underneath the renal capsule of a nude mouse the next day, For the secondary renal capsule transplantation, primary grafts were digested with $1 \mathrm{X}$ collagenase/hyaluronidase solution for $90 \mathrm{~min}$ and $0.25 \%$ Trypsin/EDTA for $6 \mathrm{~min}$ at $37^{\circ} \mathrm{C}$. Lineage-Epcam ${ }^{+} \mathrm{Zeb}^{+}$or Lineage ${ }^{-}$ Epcam $^{+}$Zeb1 $^{-}$cells were sorted via FACS and then mixed with UGM stromal cells. All serial transplantation grafts were harvested at 8 weeks after implantation. UGS from Zeb1 ${ }^{-/-}$and Zeb1 ${ }^{+/+}$ embryos were implanted under the renal capsule of male nude mice. The transplants were collected at 20 days post implantation and sectioned for further analysis.

\section{Cell lineage tracing}

To trace the fate of Zeb1 ${ }^{+}$basal cells, Zeb1-CreERT2/tdTomato mice were administrated with $1.25 \mathrm{mg}$ tamoxifen $(40 \mathrm{mg} / \mathrm{mL}$ solution, dissolved into corn oil, Sigma) via intraperitoneal injection at postnatal day 3. Two days and 12 days after the induction, prostates were collected and sectioned for immunofluorescence staining of different prostate epithelial cell markers.

\section{Organoid culture}


We utilized a previously reported protocol to culture organoids in vitro and perform organoid frozen sections for immunostaining analysis ${ }^{10}$. FACS purified Zeb $1{ }^{+}$Basal (Lineage-Sca- $1^{+}$CD $49 \mathrm{f}^{\text {hi }}$

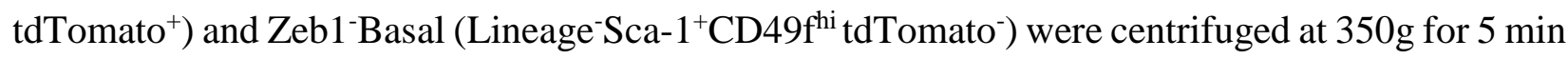
at $4^{\circ} \mathrm{C}$ and resuspended in complete organoid culture media with the density of 5000 to 10,000 prostate epithelial cells per $100 \mu \mathrm{L}$ media. The medium was refreshed every 3 days. After approximately 2 weeks, organoids were washed with cold PBS buffer repeatedly to remove the matrigel, collected via centrifugation at $350 \mathrm{~g}$ for $5 \mathrm{~min}$, then dissociated using $0.25 \%$ Trypsin/EDTA for $7 \mathrm{~min}$ at $37^{\circ} \mathrm{C}$. The single cells were centrifuged and resuspended in medium following the aforehand described procedure for a serial passaging. For section preparation, organoids were collected, fixed with 4\% PFA for 30min, washed with cold PBS buffer twice, then centrifugated and resuspended with $50 \mu \mathrm{L}$ collagen type I and incubated at $37^{\circ} \mathrm{C}$ for 1 hour to solidify the organoid/collagen slurry. The slurry was transferred into 4\%PFA solution for fixation, $30 \%$ sucrose for dehydration and finally O.C.T for embedding and section. Five-micrometer frozen sections were used for following immunostaining.

\section{Generation of CRISPR/Cas9 mediated Zeb1 knockout prostate organoids}

The lentiCRISPRv2-mCherry plasmid containing cas9 and Cherry fragments was purchased from Addgene (\#99154, kindly deposited by Dr.Agata Smogorzewska). Then the Cherry fragment was exchanged for GFP fragment via enzymatic digestion and connection followed by sgRNA cloning.

The single guide RNA sequences are ACTGCTTATATGTGAGCTAT in the upstream and GGAACAACCTGAAGTTGACT in the downstream of the sixth exon of the mouse Zebl gene. The Zeb1-cas9 lentivirus was transfected into primary prostate cells using a spinoculation method at 3,000rpm and room temperature for 2 hours. Cells were then transferred to a $15 \mathrm{~mL}$ tube and 
spinned down at $400 \mathrm{~g}$ for $5 \mathrm{~min}$. The pellet was resuspended with the complete organoid culture media and seeded at a density of 10,000 cells per well of a 96-well plate. After 3 weeks of culture, the organoids were harvested and dissociated with pre-warmed $0.25 \%$ Trypsin-EDTA. Single cell suspension was stained with anti-EpCAM antibody. $\mathrm{EpCAM}^{+} \mathrm{GFP}^{+}$cells (sgRNA successfully transfected prostate epithelial cells) were FACS sorted for organoid culture. Organoids were collected when reaching $200 \mu \mathrm{m}$ in diameter, and then fixed, dehydrated and embedded with O.C.T for frozen section preparation.

\section{RNA extraction and quantitative-PCR analysis} synthesized using the PrimeScript RT Reagent Kit (Takara). qPCR was conducted using SYBR Premix Ex Taq (Takara). Relative transcript abundance was quantified by the comparative CT method using Actin as an internal reference gene. Primers utilized in RT-PCR were shown in Supplementary Table 4.

\section{Immunofluorescence staining}

Human or mouse prostates were fixed in $4 \%$ paraformaldehyde for $20 \mathrm{~min}$ and dehydrated overnight in $30 \%$ sucrose solution. Tissues were embedded in Optional Cutting Temperature (O.C.T.) compound and placed in a $-80^{\circ} \mathrm{C}$ refrigerator for 10 minutes or longer depending on the tissue size. Frozen sections were cut at a thickness of 6 um. Sections were washed with PBS buffer and placed into $0.01 \mathrm{M}$ sodium citrate (PH 6.0) buffer for heat-induced antigen retrieval. Slides were then subjected to a blocking step in $10 \%$ donkey serum solution for $1 \mathrm{hr}$ at room temperature. 
Primary antibodies, diluted in $1 \%$ donkey serum, were added to sections overnight at $4^{\circ} \mathrm{C}$. After thorough wash with PBS buffer, secondary antibodies were applied and incubated for $1 \mathrm{hr}$ at room temperature. Sections were rinsed at least three times with PBS before being mounted by Vector Shield mounting medium containing DAPI. Antibodies used in the study is listed in Supplementary Table 5 .

\section{Immunohistochemistry}

Paraffin-embedded prostate tissue sections were deparaffinized and rehydrated following conventional methods. Harris modified hematoxylin solution (Sigma) was applied to sections for 5 min followed by water washing. Then $1 \%$ Eosin solution (Sigma) was applied to sections for 3 min. After thorough wash, sections were mounted using neutral balsam.

\section{Castration and androgen replacement}

Zeb1 reporter mice were surgically castrated. Three weeks later, mice were given dihydrotestosterone (MCE, HY-A0120) dissolved in sterile corn oil via intraperitoneal injection twice a day $(50 \mathrm{ug} / \mathrm{d})$ for prostate regeneration. Prostate collected at indicated regeneration stages (regressed, regenerated, and recovered stages post testosterone administration) for analysis.

\section{Statistical analysis}

We used the ImageJ 1.46 r software to quantify positive stained cells. All statistical analysis was evaluated using a two-tailed Student's T-test and a p-value $<0.05$ was defined to be significant. 
Data and materials availability: The single cell RNA-seq raw data used for this study are available at the GEO web with the accession number GSE111429. Codes used are deposited to the website https://github.com/HelenHeZhu/StemCell. All other data is available in the main text or the supplementary materials. 